Geometry $\&$ Topology

Volume 9 (2005) 699-755

Published: 28 April 2005

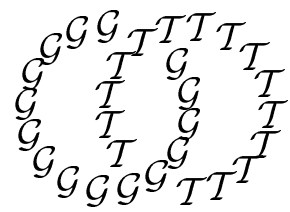

\title{
Complete intersection singularities of splice type as universal abelian covers
}

\author{
Walter D NeumanN \\ JONATHAN WAHL \\ Department of Mathematics, Barnard College, Columbia University \\ New York, NY 10027, USA \\ Department of Mathematics, The University of North Carolina \\ Chapel Hill, NC 27599-3250, USA \\ Email: neumann@math.columbia.edu and jmwahl@email.unc.edu
}

\begin{abstract}
It has long been known that every quasi-homogeneous normal complex surface singularity with $\mathbb{Q}$-homology sphere link has universal abelian cover a Brieskorn complete intersection singularity. We describe a broad generalization: First, one has a class of complete intersection normal complex surface singularities called "splice type singularities," which generalize Brieskorn complete intersections. Second, these arise as universal abelian covers of a class of normal surface singularities with $\mathbb{Q}$-homology sphere links, called "splice-quotient singularities." According to the Main Theorem, splice-quotients realize a large portion of the possible topologies of singularities with $\mathbb{Q}$-homology sphere links. As quotients of complete intersections, they are necessarily $\mathbb{Q}$-Gorenstein, and many $\mathbb{Q}$ Gorenstein singularities with $\mathbb{Q}$-homology sphere links are of this type. We conjecture that rational singularities and minimally elliptic singularities with $\mathbb{Q}$-homology sphere links are splice-quotients. A recent preprint of T Okuma presents confirmation of this conjecture.
\end{abstract}

AMS Classification numbers Primary: 32S50, 14B05

Secondary: 57M25, 57N10

Keywords: Surface singularity, Gorenstein singularity, rational homology sphere, complete intersection singularity, abelian cover

Proposed: Robion Kirby

Seconded: Ronald Fintushel, Ronald Stern
Received: 31 October 2004 Revised: 18 April 2005 


\section{Introduction}

The possible topologies for a normal singularity of a complex surface are classified (eg, 6]), but it is very rare that much is known about possible analytic types for given topology. Locally, the topology is the cone on an oriented 3-manifold $\Sigma$, called the link of the singularity. Via the configuration of exceptional curves on a good resolution $(\tilde{Y}, E) \rightarrow(Y, o)$, one can construct $\Sigma$ via plumbing according to the negative-definite dual resolution graph $\Gamma$. In this paper we will restrict to the case $\Sigma$ is a rational homology sphere, or $\mathbb{Q H S}$, ie, one for which $H_{1}(\Sigma ; \mathbb{Z})$ is finite; equivalently, the exceptional configuration is a tree of smooth rational curves.

The universal abelian covering $\tilde{\Sigma} \rightarrow \Sigma$ is finite, and can be realized by a finite map of germs $(X, o) \rightarrow(Y, o)$; the covering (or discriminant) group $H_{1}(\Sigma)$ is easily computed from the dual graph $\Gamma$. Given $\Sigma$, or (equivalently, by [6]) a graph $\Gamma$, our goal is to construct an explicit singularity $(X, o)$ whose link is $\tilde{\Sigma}$, and an action of the discriminant group which is free off $o$, so that the quotient $(Y, o)$ has graph $\Gamma$. We will achieve this under certain conditions on $\Gamma$ (Theorem [7.2).

Suppose first that $\Sigma$ is Seifert fiberable. Then it has been known for some time that the universal abelian cover of $\Sigma$ is diffeomorphic to the link of a Brieskorn complete intersection singularity $1[6,6])$. Thus a possible analytic type is as an abelian quotient of a Brieskorn complete intersection. From another point of view, consider a quasi-homogeneous $(Y, o)$ with $\mathbb{Q} H S$ link; the resolution diagram is star-shaped, and from it one can read off easily the data needed to write down a Brieskorn complete intersection and a diagonal action of the discriminant group. A look at the Seifert data shows that the quotient has the same topology as $(Y, o)$. As a bonus, one can even arrange to recover the analytic type of $Y$, because one knows the exact ingredients needed to make a quasi-homogeneous singularity.

At this point, to handle more general links, one might wonder what kinds of equations could generalize Brieskorn complete intersections.

Three-manifold theory gives a natural minimal decomposition of $\Sigma$ along embedded tori into pieces that are Seifert fiberable (a version of the JSJ-decomposition, $[8]$ ). When this set of tori is empty, $\Sigma$ is the only piece in the decomposition - the aforementioned Seifert case. More generally, associated to the JSJ

\footnotetext{
${ }^{1}$ In this paper diffeomorphisms are always assumed to preserve orientation; and, since we are interested in singularities, complete intersections are local complete intersections in the usual sense - eg, 2], p. 185.
} 
decomposition of $\Sigma$ is a certain weighted tree called a splice diagram. This is a tree with no valence two vertices, and for each node (vertex of valence $\geq 3$ ) it has a positive integer weight associated with each incident edge. The pieces in the JSJ decomposition of $\Sigma$ are in one-one correspondence with the nodes of the splice diagram. The splice diagram does not necessarily determine $\Sigma$ but it does determine its universal abelian cover.

As indicated, the link $\Sigma$ determines the topology of the minimal good resolution of the singularity, and the splice diagram $\Delta$ can easily be computed from the resolution dual graph $\Gamma$ (see, eg, Section 12). $\Delta$ has the same general shape as $\Gamma$, but degree two vertices are suppressed. Resolution graphs satisfy a negative definiteness condition which translates into a "positive edge determinants" condition for the splice diagrams of singularities.

Under a certain natural condition on $\Delta$, called the "semigroup condition," we associate a collection of $t-2$ equations in $t$ variables, where $t$ is the number of leaves of $\Delta$. There is some choice allowed in these "splice diagram equations." They generalize Brieskorn complete intersections as follows. A Brieskorn complete intersection, corresponding to a splice diagram with a single node of valence $t$, is defined by a system of $t-2$ weighted homogeneous equations. For a splice diagram with more than one node, one associates to each node a collection of $\delta-2$ equations ( $\delta$ the valence of the node) which are weighted homogeneous with respect to a system of weights associated to the node. This gives a total of $t-2$ equations. (We also allow higher weight perturbations of these equations.)

We also formulate the "congruence conditions," which depend on $\Gamma$, which guarantee that the discriminant group of the resolution acts on a set of splice diagram equations for $\Delta$. Our main results (Theorems 2.6 and Theorem [7.2) can be summarized:

Theorem The splice diagram equations associated to a splice diagram $\Delta$ with semigroup condition always describe a normal complete intersection singularity.

If splice diagram equations have been chosen equivariantly with respect to the action of the discriminant group for $\Gamma$, then the action is free away from the singular point, the quotient is a singularity with resolution graph $\Gamma$ (and hence with link $\Sigma$ ), and the covering is the universal abelian cover.

Thus for a large family of topologies - those that satisfy the semigroup and congruence conditions - we find explicit (and attractive) analytic descriptions of singularities with the given topology. Put another way, in such cases we can 
write down explicit equations for singularities with given topology (of course, modulo writing down invariants for the group action).

As finite quotients of complete intersections, these "splice-quotient singularities" are necessarily $\mathbb{Q}$-Gorenstein. Although splice diagram equations depend on choice of certain "admissible monomials," the family of analytic types for the resulting splice-quotient singularities is independent of these choices (Theorem 10.1). We had earlier (rashly) conjectured that every $\mathbb{Q}$-Gorenstein singularity with $\mathbb{Q} H S$ link should be a splice-quotient, and though this appears to be true surprisingly often (eg, quasihomogeneous singularities [7] and quotient-cusps [10]), counterexamples are now known [5]. In fact, weakly equisingular deformations (in the sense of weak simultaneous resolution) of a splice-quotient need not be of that type. Nevertheless, we conjectured in the original version of this paper that rational singularities and minimally elliptic singularities with $\mathbb{Q H S}$ link should be splice quotients (Conjecture 11.3). The recent preprint 13, of T. Okuma now offers a proof of this Conjecture (see Section 13).

In the important case that $\Sigma$ is a $\mathbb{Z}$-homology sphere (these are classified by splice diagrams with pairwise prime weights at each node), no abelian quotient is needed; so, the semigroup condition then implies one can write down directly complete intersection splice equations with given topology. We know of no complete intersection with $\mathbb{Z}$-homology sphere link which does not satisfy the semi-group conditions, or is not of splice type. (See [11.)

The leaves of the splice diagram correspond to knots in $\Sigma$, and we show that they are cut out (in the universal abelian cover) by setting the corresponding variable equal to zero. In [11] we show in the $\mathbb{Z}$-homology sphere case that the existence of functions cutting out these knots is equivalent to the singularity being of splice type, and we conjecture that this holds more generally. This point of view is useful beyond the question of existence of singularities with given topologies - it applies as well to analytic realization of germs of curves in complex surfaces (especially with $\mathbb{Z}$-homology sphere links). For instance, embedded resolution of a plane curve singularity gives rise to a (non-minimal) $\Gamma$ and $\Delta$, and one writes down an explicit equation of the curve by setting a variable equal to 0 , as in [1], Section 5 . More generally, consider the following illustration. Let $X$ be the Brieskorn variety $x^{2}+y^{3}+z^{13}=0$, and let $K$ be the knot in its link cut out by $z=0$ (this is the degree 13 fiber of the Seifert fibration of $\Sigma$ ). Form $K(p, q)$, the $(p, q)$-cable on $K$, a new knot on $\Sigma$. Then the positive edge determinant condition says that $K(p, q)$ is the link of a complex curve through the origin in $X$ if and only if $13 q>6 p$, and the semigroup condition says that this curve can be cut out by a single equation 
$f(x, y, z)=0$ if (and in this case only if) $q \geq 2$. We will return to this theme elsewhere.

Let us explain the steps needed to get to the main result. First, given a splice diagram $\Delta$ satisfying the semigroup conditions, we write down an explicit set of equations, and our first goal is to show (Theorem [2.6) that these splice diagram equations define an isolated complete intersection singularity. Every node of $\Delta$ defines a weight filtration, and one needs to prove that each associated graded is a reduced complete intersection, defined by the leading forms of the given equations. This step (Section 3) involves understanding curves defined by analogs of splice diagram equations and some detailed combinatorics involving the diagram weights. Then, to show the singularity is isolated (Section 44), one does a weighted blow-up at an "end-node" of $\Delta$, and examines singularities along the exceptional fiber. The key is to show one now has equations for a "smaller" $\tilde{\Delta}$, which by induction has an isolated singularity; a difficulty is that the new weights are related to the old ones in a rather complicated way.

We next consider the resolution diagram $\Gamma$. The discriminant group $D(\Gamma)$ is computed, and shown to act naturally (and without pseudoreflections) on $\mathbb{C}^{t}$, the space on which the splice diagram equations for $\Delta$ are defined (Section 5 ). To proceed, one needs to be able to choose splice diagram equations on which $D(\Gamma)$ acts equivariantly; this is the "congruence condition" which we need. In particular, if the semigroup and congruence conditions are satisfied for $\Gamma$, then we have an action of the discriminant group on the splice diagram equations. Our main theorem, Theorem 7.2 asserts that the quotient map is the universal abelian cover of a normal singularity whose resolution dual graph is $\Gamma$.

Finally, we have to prove Theorem 7.2 which we do by induction on the number of nodes. A key point is to explicitly lift the generators of $D(\Gamma)$ to a weighted blow-up of the singularity at an "end-node". We then have to identify the exceptional fiber and singularities that arise after factoring by the lifted group. We show that at the "worst" singular points one has (after analytic change of coordinates) a splice-quotient for a subgraph $\tilde{\Gamma}$ of $\Gamma$. Again, this involves some complicated numerics, some of which are proved in the Appendix. The one-node case is done in Section 8 and the inductive step in Section 9

We also show, as part of the main theorem, that for any node of the splice diagram, the grading on the splice-quotient induced by the weight filtration on $\mathbb{C}^{t}$ is, up to a multiple, just order of vanishing on the corresponding curve of the resolution. Using this we can deduce (Theorem 10.1) that the concept of splice-quotient singularity is a canonical concept (independent of choices). 
In Section 11] we take a two-node minimal resolution graph, and write down explicitly the semigroup and congruence conditions.

The first Appendix (Section 12) proves some results about resolution and splice diagrams that are needed in the paper, as well as a topological description of splice diagrams.

A second Appendix, added April 2005, discusses Okuma's recent preprint on the conjecture that rational and $\mathbb{Q}$ HS-link minimally elliptic singularities are always splice quotients.

Acknowledgements The first author's research is supported under NSF grant DMS-0083097 and the second author's under NSA grant MDA904-021-0068.

\section{Semigroup conditions and splice equations}

We first recall the concept of "splice diagram," a certain kind of weighted tree. Given a finite tree, the valency of a vertex is the number of incident edges. A node is a vertex of valency $\geq 3$ and a leaf is a vertex of valency 1 .

A splice diagram $\Delta$ is a finite tree with no valence 2 vertices, decorated with integer weights as follows: for each node $v$ and edge $e$ incident at $v$ an integer weight $d_{v e}$ is given (see Section 12 for examples and more detail). Thus an edge joining two nodes has weights associated to each end, while an edge from a node to a leaf has just one weight at the node end. The edge determinant of an edge joining two nodes is the product of the two weights on the edge minus the product of the weights adjacent to the edge. Splice diagrams that arise in the study of links of complex singularities always satisfy the following conditions:

- All weights are positive.

- All edge determinants are positive.

- One has the "ideal condition" on weights (see below).

To explain the third of these, we need more notation.

Notation 2.1 For a node $v$ and an edge $e$ at $v$, let $d_{v e}$ be the weight on $e$ at $v$, and $d_{v}$ the product of all edge-weights $d_{v e}$ at $v$. For any pair of distinct vertices $v$ and $w$, let $\ell_{v w}$ (the linking number) be the product of all the weights adjacent to, but not on, the shortest path from $v$ to $w$ in $\Delta$. Define 
$\ell_{v w}^{\prime}$ similarly, except one excludes weights around $v$ and $w$. (Thus $\ell_{v w}^{\prime}=1$ if $v$ and $w$ are adjacent, and $\ell_{v w}=\ell_{v w}^{\prime}$ if $v$ and $w$ are both leaves.) Finally, let $\Delta_{v e}$ be the subgraph of $\Delta$ cut off from $v$ by $e$ (ie, on the " $e$-side of $v$ ").

Definition 2.2 Ideal Condition For each node $v$ and adjacent edge $e$ of $\Delta$, the edge-weight $d_{v e}$ is divisible by the GCD of all $\ell_{v w}^{\prime}$ with $w$ a leaf of $\Delta$ in $\Delta_{v e}$; in other words, $d_{v e}$ is in the following ideal of $\mathbb{Z}$ :

$$
d_{v e} \in\left(\ell_{v w}^{\prime}: w \text { a leaf of } \Delta \text { in } \Delta_{v e}\right) .
$$

We are interested in splice diagrams that satisfy the stronger condition:

Definition 2.3 Semigroup Condition The semigroup condition says that for each node $v$ and adjacent edge $e$ of $\Delta$, the edge-weight $d_{v e}$ is in the following sub-semigroup of $\mathbb{N}$ :

$$
d_{v e} \in \mathbb{N}\left\langle\ell_{v w}^{\prime}: w \text { a leaf of } \Delta \text { in } \Delta_{v e}\right\rangle .
$$

For each edge $e$ at $v$ the semigroup condition lets us write

$$
d_{v e}=\sum_{w \text { a leaf in } \Delta_{v e}} \alpha_{v w} \ell_{v w}^{\prime}, \quad \text { with } \alpha_{v w} \in \mathbb{N} \cup\{0\} .
$$

It is easy to see that this is equivalent to

$$
d_{v}=\sum_{w} \alpha_{v w} \ell_{v w}
$$

Assume from now on that $\Delta$ satisfies the semigroup condition. To each leaf $w$ we associate a variable $z_{w}$.

Definition 2.4 $v$-weighting; admissible monomials Fix a node $v$. Then the $v$-weighting, or $v$-filtration, of the polynomial ring in the $z_{w}$ 's is defined by assigning weight $\ell_{v w}$ to $z_{w}$.

An admissible monomial (associated to the edge $e$ at the node $v$ ) is a monomial $M_{v e}=\prod_{w} z_{w}^{\alpha_{v w}}$, the product over leaves $w$ in $\Delta_{v e}$, with exponents satisfying the above equations (11). In particular, each admissible monomial $M_{v e}$ is $v$ weighted homogeneous, of total $v$-weight $d_{v}$.

Definition 2.5 Splice diagram equations Let $\Delta$ be a splice diagram with $t$ leaves satisfying the semigroup condition. To each leaf $w$ associate a variable $z_{w}$; for each node $v$ and adjacent edge $e$, choose an admissible monomial $M_{v e}$. 
Then splice diagram equations for $\Delta$ consist of a collection of equations of the form

$$
\sum_{e} a_{v i e} M_{v e}+H_{v i}=0, \quad v \text { a node }, i=1, \ldots, \delta_{v}-2
$$

where

- for every $v$, all maximal minors of the $\left(\left(\delta_{v}-2\right) \times \delta_{v}\right)$-matrix $\left(a_{v i e}\right)$ have full rank

- $H_{v i}$ is a convergent power series in the $z_{w}$ 's all of whose monomials have $v$-weight $>d_{v}$.

It is easy to see one has exactly $t-2$ equations in the $t$ variables. The corresponding subscheme $X(\Delta) \subset \mathbb{C}^{t}$ is a splice diagram surface singularity.

Theorem 2.6 Let $X=X(\Delta) \subset \mathbb{C}^{t}$ be a splice diagram surface singularity. Then:

(1) $X$ is a two-dimensional complete intersection with an isolated singularity at the origin.

(2) For any node $v$, the corresponding weight filtration has associated graded ring a reduced complete intersection, defined by the $v$-leading forms of the splice equations.

The theorem will be proved partly by induction on the number of nodes of $\Delta$. Once we know the singularities are isolated, one can recover all the analytic types by restricting to polynomials $H_{v i}$ in the definition. We allow splice diagrams in which an edge-weight leading to a leaf may be 1, as the "minimality" assumption which avoids this could be lost in our inductive process.

One could define a more general class of equations by allowing, for each node $v$ and edge $e$, linear combinations of admissible monomials, rather than multiples of a fixed one. It follows from the theorem (and proof) that for generic coefficients, these give isolated singularities with the same properties. But, in the situation of most interest to us here (Theorem [7.2), where the monomial $M_{v e}$ also satisfies an equivariance condition, this is not a generalization, since we will show (Theorem 10.1) that any other allowed monomial $M_{v e}^{\prime}$ for $v, e$ then differs from some multiple of $M_{v e}$ by something of higher weight.

Given that the maximal minors of the coefficient matrix $\left(a_{v i e}\right)$ have full rank, one may apply row operations to the matrix (which is the same as taking linear 
combinations of the corresponding equations), to put the $\left(\delta_{v}-2\right) \times \delta_{v}$ coefficient matrix in the form

$$
\left(\begin{array}{cccccc}
1 & 0 & \ldots & 0 & a_{1} & b_{1} \\
0 & 1 & \ldots & 0 & a_{2} & b_{2} \\
\vdots & \vdots & \ddots & \vdots & \vdots & \vdots \\
0 & 0 & \ldots & 1 & a_{\delta_{v}-2} & b_{\delta_{v}-2}
\end{array}\right)
$$

with $a_{i} b_{j}-a_{j} b_{i} \neq 0$ for all $i \neq j$, and all $a_{i}$ and $b_{i}$ nonzero. We will often assume we have done so. In this way, the defining equations are sums of three monomials, plus higher order terms. (Sometimes - for instance the next section - it will be more convenient to move the last two columns of the above matrix to the first two columns.)

\section{Splice diagram curves and associated gradeds}

As in [1], one can define curves using a modified version of splice diagrams. Let $\left(\Delta, w^{\prime}\right)$ be a splice diagram with distinguished leaf $w^{\prime}$. But now, at any node, the edge weight in the direction of $w^{\prime}$ is irrelevant and should be omitted or ignored. $\left(\Delta, w^{\prime}\right)$ satisfies the semigroup condition if for every node $v$ and adjacent edge $e$ pointing away from $w^{\prime}$, the edge-weight $d_{v e}$ is in the semigroup generated by all the $\ell_{v w}^{\prime}$ with $w$ in $\Delta_{v e}$. This condition is automatic if $\Delta$ is a splice diagram that satisfies the semigroup condition and $\left(\Delta, w^{\prime}\right)$ is obtained by ignoring edge weights in the direction of $w^{\prime}$.

As before, associate a variable $z_{w}$ to each leaf different from $w^{\prime}$; and, for every node $v$ and adjacent edge $e$ pointing away from $w^{\prime}$, choose an admissible monomial $M_{v e}$. One can define a splice diagram curve $C=C\left(\Delta, w^{\prime}\right)$ via equations for all nodes $v$ :

$$
\sum_{e} a_{v i e} M_{v e}=0, \quad i=1, \ldots, \delta_{v}-2,
$$

where for all $v$ the $\left(\delta_{v}-2\right) \times\left(\delta_{v}-1\right)$ matrix $\left(a_{v i e}\right)$ is required to have full rank. (Note that we now don't allow higher order terms.) Enumerating the admissible monomials at $v$ as $M_{v j}, 1 \leq j \leq \delta_{v}-1$, one can always write the system of equations as

$$
M_{v j}=a_{v j} M_{v 1}, \quad j=2, \ldots, \delta_{v}-1,
$$

where all $a_{v j} \neq 0$. Assign to the variable $z_{w}$ the weight $\ell_{w^{\prime} w}$; then the equations at $v$ are weighted homogeneous, of total weight $\ell_{w^{\prime} v}$.

Theorem 3.1 Let $C$ be a splice diagram curve as above. 
(1) At a point of $C$ for which one coordinate is 0 , all coordinates are 0 .

(2) Except at the origin, $C$ is a smooth curve.

(3) The number of components of $C$ is the GCD of the weights of the variables.

(4) $C$ is a reduced complete intersection curve, and every irreducible component is isomorphic to a monomial curve.

(5) For each leaf $w \neq w^{\prime}$, let $\alpha_{w}$ be an integer $\geq 0$, not all equal 0 . Then for $b \neq 0$, the intersection $C \cap\left\{\prod z_{w}^{\alpha_{w}}=b\right\}$ is everywhere transverse and consists of $\sum \alpha_{w} \ell_{w^{\prime} w}$ points.

Proof Denote by $v^{\prime}$ the node adjacent to the distinguished leaf $w^{\prime}$.

One can use induction in several ways: for instance, remove $v^{\prime}$ from the diagram, then reinsert a distinguished leaf in its place for $\delta_{v^{\prime}}-1$ splice subdiagrams pointing away from $w^{\prime}$. The splice type equations for the subdiagrams are among the equations we started with. Combining with the equations at $v^{\prime}$, one easily gets the first claim.

For the second assertion, it is easier to use a different induction. Let $v$ be an endnode, adjacent to $\sigma:=\delta_{v}-1$ leaves, say $w_{1}, \ldots, w_{\sigma}$, with associated coordinates $z_{1}, \ldots, z_{\sigma}$. Then some splice equation at $v$ is of the form $z_{2}^{m_{2}}-a z_{1}^{m_{1}}=0$ $(a \neq 0)$; this polynomial has $k$ irreducible factors, where $k$ is the GCD of $m_{1}$ and $m_{2}$. In a neighborhood of the point in question, exactly one of these factors vanishes, so we can solve $z_{1}=t^{m_{2} / k}, z_{2}=a^{\prime} t^{m_{1} / k}$. Plug these into all the remaining equations. One then recognizes splice diagram equations associated to a diagram with one less leaf. When $\sigma \geq 3$, replace $w_{1}$ and $w_{2}$ by one leaf, with edge-weight $m_{1} m_{2} / k$, and then divide all edge weights pointing towards the node $v$ by $h$ (they are divisible by $k$, by the semigroup condition). When $\sigma=2$, drop the two leaves, making $v$ into a new leaf, and again divide the same edge-weights by $k$. It is straightforward to check that one has equations associated to a new splice diagram. This process gives the inductive step necessary to prove the claim.

The third assertion uses the same induction. Number the leaves $w_{1}, \ldots, w_{\tau}$ and abbreviate the weight $\ell_{w^{\prime} w_{i}}$ of the $i$-th variable by $\ell_{i}$. Then the weights of the variables in the new splice diagram in the induction are $\ell:=\ell_{1} / m_{2}=\ell_{2} / m_{1}$, and $\ell_{3} / k, \ldots, \ell_{\tau} / k$. The inductive step thus follows from the equation

$$
\operatorname{gcd}\left(m_{1} \ell, m_{2} \ell, \ell_{3}, \ldots, \ell_{\tau}\right)=k \cdot \operatorname{gcd}\left(\ell, \ell_{3} / k, \ldots, \ell_{\tau} / k\right) .
$$

Since $C$ is a curve with one singularity, and is defined by the appropriate number of equations, it is a complete intersection (necessarily reduced). Since $C$ is 
weighted homogeneous, it has a $\mathbb{C}^{*}$-action. Any irreducible component still has a $\mathbb{C}^{*}$-action, hence is a monomial curve. In fact, let $h$ denote the GCD of all the weights $\ell_{i}$ of the variables, and consider "reduced weights" $\bar{\ell}_{i}:=\ell_{i} / h$. Then for any $\left(p_{1}, \ldots, p_{\tau}\right) \neq(0, \ldots, 0)$ on $C$, one sees that $\left(z_{1}, \ldots, z_{\tau}\right)=\left(p_{1} t^{\bar{\ell}_{1}}, \ldots, p_{\tau} t^{\bar{\ell}_{\tau}}\right)$ defines an irreducible monomial curve contained in $C$ through the point, hence gives an irreducible component.

As for the last count of solutions, the function $f:=\prod z_{w}^{a_{w}}$, restricted to the component $\left(p_{1} t^{\bar{\ell}_{1}}, \ldots, p_{\tau} t^{\bar{\ell}_{\tau}}\right)$ of $C$, has the form $p t^{\sum a_{i} \bar{\ell}_{i}}$ for some $p \neq 0$, so it has exactly $\sum a_{i} \bar{\ell}_{i}$ inverse images over any $b \neq 0$. Thus, the intersection of $C$ with $f\left(z_{1}, \ldots, z_{\tau}\right)=b$ is transversal for any $b \neq 0$ and the number of intersection points is $\sum a_{i} \bar{\ell}_{i}$ on each of the $h$ components of $C$, so there are $h \sum a_{i} \bar{\ell}_{i}=\sum a_{i} \ell_{i}$ intersection points in all.

From the preceding result one derives the second part of Theorem 2.6]concerning $X(\Delta)$.

Proof of Theorem 2.6 (2) Enumerate the chosen admissible monomials at $v$ as $M_{i}, 1 \leq i \leq \delta_{v}$; these are weighted homogeneous of degree $d_{v}$ with respect to the $v$-weighting. So the leading forms of the equations at $v$ may be written

$$
M_{i}-a_{i} M_{1}-b_{i} M_{2}=0, \quad 3 \leq i \leq \delta_{v} .
$$

Lemma 3.2 At a node $v^{\prime} \neq v$, consider an admissible monomial $M_{v^{\prime} e}$.

(1) If e does not point towards $v$, then the $v$-weight of $M_{v^{\prime} e}$ is $\ell_{v^{\prime} v}$.

(2) If e points towards $v$, then the $v$-weight of $M_{v^{\prime} e}$ is strictly greater than $\ell_{v^{\prime} v}$.

(3) If a monomial has $v^{\prime}$-weight $>d_{v^{\prime}}$, then its $v$-weight is strictly greater than $\ell_{v^{\prime} v}$.

Proof (Cf. Theorem 7.3 in [1].) One checks directly that for any $w$

$$
\frac{\ell_{v w}}{\ell_{v^{\prime} w}}=\frac{\ell_{v^{\prime} v}}{d_{v^{\prime}}} D_{e_{1}^{\prime}} \ldots D_{e_{k}^{\prime}}
$$

where:

- $e_{1}^{\prime}, \ldots, e_{k}^{\prime}$ are the edges that are on the path from $v^{\prime}$ to $v$ but not on the path from $w$ to $v$;

- for any edge $e, D_{e}$ is the product of the edge weights on $e$ divided by the product of edge weights directly adjacent to $e$ (so $D_{e}>1$ by the edge determinant condition). 
Thus $\ell_{v w} / \ell_{v^{\prime} w}$ takes its minimum value (namely $\ell_{v^{\prime} v} / d_{v^{\prime}}$ ) if and only if $w$ is beyond $v^{\prime}$ from the point of view of $v$. It follows that the admissible monomials at $v^{\prime}$ all have the same $v$-weight except for the near monomial for $v$, which has higher $v$-weight. One has the last assertion of the lemma as well.

It follows from the lemma that the $v$-leading form of a splice diagram equation at $v^{\prime}$ is given by the combination of the admissible monomials pointing away from $v$; all other terms have higher $v$-weight. We prove that these equations, plus the ones at $v$, together define a two-dimensional complete intersection, whence they define the full associated graded ring associated to the $v$-filtration.

The vertices $\neq v$ of $\Delta$ divide into $\delta_{v}$ groups, depending upon the $\Delta_{v e}$ to which a $v^{\prime}$ belongs. In a sector consisting of more than one leaf, consider the $v$-leading forms of the splice equations corresponding to vertices in that sector. Using Lemma 3.2 these forms can be seen to provide a complete set of splice diagram curve equations for the corresponding set of variables (where $v$ is viewed as a "root" of the sector). Therefore, in any sector, if one of the $z_{w}$ vanishes at a point, so do all the other leaf variables (by Theorem 3.1). In particular, at a point of vanishing of two variables, one from each of the first two edge directions, one has the vanishing as well of $M_{1}$ and $M_{2}$. This implies that every $M_{i}$ vanishes, whence one variable in every group vanishes, so every variable vanishes. Consequently, the locus of the particular $t-2$ leading forms plus these two variables is 0 -dimensional, hence must be a 0 -dimensional complete intersection. Equivalently, those leading forms and two variables form a regular sequence. Now recall the following well-known result in commutative algebra:

Lemma 3.3 Let $f_{1}, \ldots, f_{r}$ be a sequence of elements in a positively graded polynomial ring $P$, generating an ideal $I$. Suppose that the leading forms $\bar{f}_{1}, \ldots, \bar{f}_{r}$ form a regular sequence. Then the $f_{i}$ form a regular sequence, and the ideal of leading forms of $I$ is generated by the $\bar{f}_{i}$.

We conclude that the associated graded ring of the $v$-filtration of $X(\Delta)$ is a two-dimensional complete intersection, defined by the $t-2$ leading forms as above. In addition, $X(\Delta)$ is a complete intersection at the origin.

Corollary 3.4 Let $X(\Delta)$ be a splice diagram singularity as above. Then for any two variables $z, z^{\prime}$, the locus

$$
X(\Delta) \cap\left\{z=z^{\prime}=0\right\}
$$

contains the origin as an isolated point. 
Proof Choose $v$ the node adjacent to the leaf corresponding to $z$. In Spec of the associated graded, the locus $\left\{z=z^{\prime}=0\right\}$ is exactly the origin, so the associated graded modulo $z$ and $z^{\prime}$ is a 0-dimensional complete intersection. Thus, the local ring of $X$ at the origin modulo these two variables is also 0dimensional.

One can also see that any of these associated graded rings is reduced; it suffices to show generic reducedness. Choose a point all of whose coordinates are nonzero. Then in every sector, one can solve the splice equations around that point and set each variable $z$ equal to a constant times a power of a new variable $t$. This transforms that sector's admissible monomial into a power of one variable $t$. This puts the equations corresponding to $v$ into the form of a Brieskorn complete intersection. In particular, Spec of the associated graded is smooth at points for which all coordinates are non-0; the only singular points occur along the curves obtained by setting a variable equal to 0 .

To show the singularity of $X(\Delta)$ is isolated, and to get a handle on resolution diagrams, we do a weighted blow-up.

\section{Weighted blow-up and the proper transform}

Let $z_{1}, \ldots, z_{t}$ be coordinates on an affine space $\mathbb{C}^{t}$, where $z_{i}$ has positive integer weight $m_{i}$. Blowing-up the corresponding weight filtration gives the weighted blow-up $Z \rightarrow \mathbb{C}^{t}$, an isomorphism off the inverse image of the origin. $Z$ is covered by $t$ affine varieties $U_{i}$, each of which is a quotient of an affine space $V_{i}$ by a cyclic group of order $m_{i}$. $V_{1}$ has coordinates $A_{1}, \ldots, A_{t}$, related to the $z_{i}$ via

$$
z_{1}=A_{1}^{m_{1}}, z_{2}=A_{1}^{m_{2}} A_{2}, \ldots, z_{t}=A_{1}^{m_{t}} A_{t} ;
$$

$U_{1}$ equals $V_{1}$ modulo the action of the cyclic group generated by

$$
S=\left[-1 / m_{1}, m_{2} / m_{1}, \ldots, m_{t} / m_{1}\right],
$$

where we are using the notation

$$
\left[q_{1}, \ldots, q_{t}\right]:=\left(\exp \left(2 \pi i q_{1}\right), \ldots, \exp \left(2 \pi i q_{t}\right)\right) .
$$

Consider now a splice diagram singularity $X=X(\Delta)$, where as usual $t$ is the number of leaves. Let $v^{*}$ be an end-node of $\Delta$, ie, a node all but one of whose edges is adjacent to a leaf. Putting this node on the left side of $\Delta$, we write

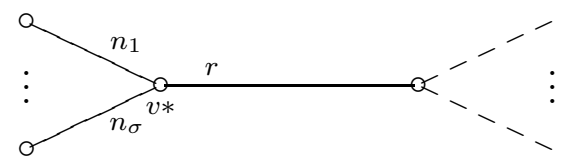


(As the one-node case is easy and is revisited below, we assume there are at least two nodes). Blow-up the corresponding weight filtration, where $z_{w}$ has weight $\ell_{v^{*} w}$. Since the associated graded of $X$ with respect to the filtration is a complete intersection, the proper transform of $X$ in $Z$ or the $V_{i}$ is defined by the proper transform of the splice equations. Further, since the origin is the only point of $X$ at which two coordinates vanish, one sees the full transform of $X$ by considering the proper transforms $X_{i}$ on $V_{i}$ for $i=1,2$.

Denote by $\sigma$ the number of leaves adjacent to $v^{*}, \tau=t-\sigma$ the number of remaining leaves and number these leaves $w_{1}, \ldots, w_{\sigma+\tau}$. Call the corresponding variables $x_{1}, \ldots, x_{\sigma}, y_{1}, \ldots, y_{\tau}$. Write $N=n_{1} n_{2} \ldots n_{\sigma}, N_{i}=N / n_{i}$. Then the weight of $x_{i}$ is $r N_{i}$, while that of $y_{k}$ is $N \ell_{k}^{\prime}$, where $\ell_{k}^{\prime}:=\ell_{v^{*} w_{\sigma+k}}^{\prime}$. We write the splice diagram equations in two groups.

First, one has

$$
x_{i}^{n_{i}}+a_{i} x_{\sigma}^{n_{\sigma}}=b_{i} \underline{\underline{\underline{\alpha}}} \underline{\underline{\alpha}}+H_{i}(\underline{x}, \underline{y}), \quad 1 \leq i \leq \sigma-1 .
$$

Here, $\underline{\underline{\alpha}} \underline{\underline{\alpha}}:=\prod_{k=1}^{\tau} y_{k}^{\alpha_{k}}$ is an admissible monomial for $v^{*}$, so $\sum \alpha_{k} \ell_{k}^{\prime}=r$. The term $\bar{H}_{i}(\underline{x}, \underline{y})$ (shorthand for $H_{i}\left(x_{1}, \ldots, x_{\sigma}, y_{1}, \ldots, y_{\tau}\right)$ ) contains monomials of higher $v^{*}$-weight.

Second, for each node $v \neq v^{*}$, order the admissible monomials $M_{v j}, j=$ $1, \ldots, \delta_{v}$ so that $M_{v 1}$ corresponds to the edge pointing in the direction of $v^{*}$ (thus only $M_{v 1}$ can involve any $x_{i}$ variables). The corresponding equations are of the form

$$
\sum_{j=1}^{\delta_{v}} a_{v i j} M_{v j}=H_{v i}(\underline{x}, \underline{y}), \quad i=1, \ldots, \delta_{v}-2 .
$$

The transforms of the first group of equations (2a) on $V_{1}$ have the form

$$
\begin{aligned}
1+a_{1} A_{\sigma}^{n_{\sigma}} & =b_{1} \underline{B}^{\underline{\alpha}}+A_{1} J_{1}(\underline{A}, \underline{B}) \\
A_{i}^{n_{i}}+a_{i} A_{\sigma}^{n_{\sigma}} & =b_{i} \underline{B}^{\underline{\alpha}}+A_{1} J_{i}(\underline{A}, \underline{B}), \quad 2 \leq i \leq \sigma-1 .
\end{aligned}
$$

For equations (2b) corresponding to another node $v$, it follows from Lemma 3.2 that the transforms of such equations take the form

$$
\sum_{j=2}^{\delta_{v}} a_{v i j} M_{v j}(\underline{B})=A_{1} H_{v i}^{\prime}(\underline{A}, \underline{B}), \quad i=1, \ldots, \delta_{v}-2 .
$$

Here, $M_{v j}$ (for $j>1$ ) is the same monomial as before, but evaluated on the $B_{k}$ instead of the $y_{k}$. These equations are obtained from the ones above by substituting for the $x_{i}$ and $y_{k}$, and then dividing through by the highest power of $A_{1}$ that occurs, which is $A_{1}^{\ell^{*} v}$ by Lemma 3.2 . 
One needs to get a handle on the singular locus of $X_{1}$. First, the exceptional divisor is the subscheme of $X_{1}$ defined by $A_{1}=0$; it is given by equations

$$
\begin{aligned}
1+a_{1} A_{\sigma}^{n_{\sigma}} & =b_{1} \underline{B}^{\underline{\alpha}}, & & \\
A_{i}^{n_{i}}+a_{i} A_{\sigma}^{n_{\sigma}} & =b_{i} \underline{B}^{\underline{\alpha}}, & & 2 \leq i \leq \sigma-1, \\
\sum_{j=2}^{\delta_{v}} a_{v i j} M_{v j}(\underline{B}) & =0, & v \neq v^{*} \text { a node, }, & i=1, \ldots, \delta_{v}-2 .
\end{aligned}
$$

Lemma 4.1 The curve defined above has singularities only at the $n_{2} \ldots n_{\sigma}$ points for which all $B_{k}=0$ (hence no $A_{i}=0$ for $i>1$ ). Every connected component of the curve contains such a point. At a point for which $A_{2}=0$, $A_{2}$ is a local analytic coordinate; there are $n_{3} \ldots n_{\sigma} r$ such points.

Proof The equations arising from $v$ different from $v^{*}$ are splice diagram curve equations for the splice diagram obtained by removing from $\Delta$ the $\sigma$ edges and leaves adjacent to $v^{*}$, which is now viewed as a root. By Theorem 3.1, these define a reduced complete intersection curve $C$ in the variables $B_{k}$, with one singularity at the origin. Adding in the first $\sigma-1$ equations (and variables $\left.A_{2}, \ldots, A_{\sigma}\right)$ defines a branched cover of this curve, which by the Jacobian criterion is unramified except when one of the $A_{i}$ is 0 . There are thus $N_{1}=n_{2} \ldots n_{\sigma}$ singular points lying above the origin. The image of any connected component under the finite cover must contain the origin, whence each component contains at least one of the $N_{1}$ points.

Next consider a point where $A_{2}=0$. The genericity condition on the coefficients implies all other $A_{i} \neq 0$. Some $B_{j} \neq 0$, hence all $B_{k} \neq 0$, by Theorem 3.1 In a neighborhood of such a point, we can write $B_{i}=c_{i} t^{\ell_{i}}$, as in the proof of Theorem 3.1] and so replace the term $\underline{B}^{\underline{\alpha}}$ by a constant times $t^{r}$. So the curve is now defined by $\sigma-1$ equations in the variables $A_{2}, \ldots, A_{\sigma}, t$. Again the Jacobian criterion implies that $A_{2}$ is a coordinate at any point where all the other coordinates are non-zero (one needs again the precise genericity of the coefficients). To count the points we note that the value of $\underline{B}^{\underline{\alpha}}$ is determined, so part 5 of Theorem 3.1 gives us exactly $r$ points on the curve in the variables $B_{k}$, and above each of these there are $n_{3} \ldots n_{\sigma}$ points when one adds in the variables $A_{2}=0, A_{3}, \ldots, A_{\sigma}$.

Thus, the singular locus of $X_{1}$ intersects the exceptional divisor only at the $N_{1}$ singular points described above. We show these are isolated singular points of $X_{1}$. Repeating for $X_{2}$ will imply that $X$ has an isolated singularity. In 
addition, we will have the necessary set-up to study singular points on $X_{1}$ and on its quotient by finite groups (as needed in Section 9).

Choose a point where $A_{1}$ and all the $B_{k}$ are 0 ; thus, all $A_{i}$ are non-zero for $i>1$. Now, the matrix of $A_{i}$-partial derivatives $(2 \leq i \leq \sigma)$ of the $\sigma-1$ equations (3a) is easily seen to be invertible at such a point. So, by the implicit function theorem one can, in a neighborhood of such a point, solve uniquely these equations, and write each $A_{i}$ as a convergent power series in $A_{1}, B_{1}, \ldots, B_{\tau}$, with non-zero constant term. Plug these convergent power series into the second group of equations (3b). We show one now has a set of splice diagram equations for a smaller diagram $\tilde{\Delta}$, which by induction represents an isolated singularity. This will complete the proof of Theorem 2.6.

Let $\tilde{\Delta}$ be the splice diagram whose underlying graph is $\Delta$ less the $\sigma$ edges and leaves adjacent to $v^{*}$; thus $v^{*}$ is now replaced by a leaf $w^{*}$. Edge-weights not pointing towards $v^{*}$ are defined to be the same as before. For a node $v$ and the edge at $v$ pointing towards $v^{*}$, define a new edge-weight by

$$
\tilde{d}_{v 1}=r d_{v 1}-N\left(d_{v} / d_{v 1}\right)\left(\ell_{v v^{*}}^{\prime}\right)^{2}
$$

This is an integer, and its positivity is easily seen by multiplying all the edgedeterminant inequalities between $v^{*}$ and $v$. One readily checks by induction over distance of an edge from $v^{*}$ that each edge determinant of $\tilde{\Delta}$ is $r$ times the corresponding edge-determinant of $\Delta$, hence positive.

Lemma 4.2 Assign the variables $A_{1}$ to $w^{*}$ and $B_{k}$ to corresponding other leaves of $\tilde{\Delta}$. Then the proper transforms of the second group of equations for $\Delta$, with substitutions for $A_{2}, \ldots, A_{\sigma}$, are (in a neighborhood of the point in question) splice diagram equations for $\tilde{\Delta}$ (which in particular must satisfy the semigroup condition).

Proof Choose a node $v$ of $\tilde{\Delta}$. Since the edge-weights of $\tilde{\Delta}$ are the same as the corresponding ones of $\Delta$ except on edges pointing to $w^{*}$, one sees that the old admissible monomials $M_{v i}(\underline{y})$ for $i>1$ and $\Delta$ yield admissible monomials $M_{v i}(\underline{B})$ for $\tilde{\Delta}$. One must check the replacement for $M_{v 1}(\underline{x}, \underline{y})$ in the new equations.

More generally, consider any monomial

$$
\prod_{i} x_{i}^{\beta_{i}} \prod_{k} y_{k}^{\gamma_{k}}
$$

appearing in an equation associated to the node $v$. Going up to $X_{1}$ means substituting for $x_{i}$ and $y_{k}$ in terms of the $A_{i}$ and $B_{k}$; taking proper transform 
means subtracting $\ell_{v^{*} v}$ from the exponent of $A_{1}$. This gives a monomial of the form

$$
A_{1}^{Q} A_{2}^{\beta_{2}} \ldots A_{\sigma}^{\beta_{\sigma}} \prod B_{k}^{\gamma_{k}}
$$

Here, one has

$$
Q=r \sum \beta_{i} N_{i}+N \sum \gamma_{k} \ell_{k}^{\prime}-\ell_{v v^{*}}
$$

In a neighborhood of the singular point, $A_{i}$ for $i>1$ is a power series in the variables $A_{1}, B_{k}$ with non-zero constant term; so we are really considering (up to a fixed factor plus higher-order terms) the transformed monomial

$$
A_{1}^{Q} \prod B_{k}^{\gamma_{k}}
$$

If the original monomial is the particular admissible $M_{v 1}$ for $\Delta$, one has

$$
\sum_{i=1}^{\sigma} \beta_{i} \ell_{v w_{i}}+\sum_{k=1}^{\tau} \gamma_{k} \ell_{v w_{\sigma+k}}=d_{v}
$$

the second sum being over leaves on the $v^{*}$ side of $v$. Let us put a $\sim$ over a linking number or degree computed in $\tilde{\Delta}$. To prove the transformed monomial is admissible for $\tilde{\Delta}$, one must prove

$$
Q \tilde{\ell}_{v v^{*}}+\sum_{k=1}^{\tau} \gamma_{k} \tilde{\ell}_{v w_{\sigma+k}}=\tilde{d}_{v}
$$

We claim that, in fact,

$$
Q \tilde{\ell}_{v v^{*}}+\sum_{k=1}^{\tau} \gamma_{k} \tilde{\ell}_{v w_{\sigma+k}}-\tilde{d}_{v}=r\left(\sum_{i=1}^{\sigma} \beta_{i} \ell_{v w_{i}}+\sum_{k=1}^{\tau} \gamma_{k} \ell_{v w_{\sigma+k}}-d_{v}\right),
$$

so (6) is equivalent to (5). We postpone the proof of (7), which holds even if some of the leaves $w_{\sigma+k}$ are beyond $v$ from $v^{*}$ (we need this later).

We conclude that our equations have appropriate admissible monomials; in particular, $\tilde{\Delta}$ satisfies the semigroup condition. Further, the coefficients at each node satisfy the appropriate genericity condition, since they are the same as before except for a multiple of the fixed factor just mentioned.

Next, one must show a monomial of $v$-weight $>d_{v}$ gives rise to a transformed monomial of weight $>\tilde{d}_{v}$ with respect to the new $\tilde{v}$-valuation. Thus, for non-negative integers $\beta_{i}, \gamma_{k}$, the inequality

$$
\sum_{i=1}^{\sigma} \beta_{i} \ell_{v w_{i}}+\sum_{k=1}^{\tau} \gamma_{k} \ell_{v w_{\sigma+k}}>d_{v}
$$


should imply the inequality

$$
Q \tilde{\ell}_{v v^{*}}+\sum_{k=1}^{\tau} \gamma_{k} \tilde{\ell}_{v w_{\sigma+k}}>\tilde{d}_{v} .
$$

The equivalence of these inequalities also follows from equation (77).

To complete the proof we must thus prove equation (77). First note that multiplying equation (44) by $d_{v} / d_{v 1}$ gives

$$
\tilde{d}_{v}=r d_{v}-N\left(\frac{d_{v}}{d_{v 1}} \ell_{v v^{*}}^{\prime}\right)^{2}=r d_{v}-\tilde{\ell}_{v v^{*}} \ell_{v v^{*}}
$$

Hence

$$
\begin{aligned}
& Q \tilde{\ell}_{v v^{*}}+\sum \gamma_{k} \tilde{\ell}_{v w_{\sigma+k}}-\tilde{d}_{v} \\
= & \left(r \sum \beta_{i} N_{i}+N \sum \gamma_{k} \ell_{k}^{\prime}-\ell_{v v^{*}}\right) \tilde{\ell}_{v v^{*}}+\sum \gamma_{k} \tilde{\ell}_{v w_{\sigma+k}}-\left(r d_{v}-\tilde{\ell}_{v v^{*}} \ell_{v v^{*}}\right) \\
= & r \sum \beta_{i} N_{i} \tilde{\ell}_{v v^{*}}+\sum \gamma_{k}\left(N \ell_{k}^{\prime} \tilde{\ell}_{v v^{*}}+\tilde{\ell}_{v w_{\sigma+k}}\right)-r d_{v} \\
= & r \sum \beta_{i} \ell_{v w_{i}}+r \sum \gamma_{k} \ell_{v w_{\sigma+k}}-r d_{v},
\end{aligned}
$$

where the equality $N \ell_{k}^{\prime} \tilde{\ell}_{v v^{*}}+\tilde{\ell}_{v w_{\sigma+k}}=r \ell_{v w_{\sigma+k}}$ is seen as follows: Denote by $v_{k}$ the vertex where the paths from $v$ to $v^{*}$ and $w_{\sigma+k}$ diverge (so $v_{k}=v$ iff $w_{\sigma+k}$ is beyond $v$ from $v^{*}$ ). Then

$$
\begin{gathered}
N \ell_{k}^{\prime} \tilde{\ell}_{v v^{*}}+\tilde{\ell}_{v w_{\sigma+k}}=\ell_{k}^{\prime} \ell_{v v^{*}}+\frac{\ell_{v w_{\sigma+k}}}{d_{v_{k}}} \tilde{d}_{v_{k}}=\ell_{k}^{\prime} \ell_{v v^{*}}+\frac{\ell_{v w_{\sigma+k}}}{d_{v_{k}}}\left(r d_{v_{k}}-\tilde{\ell}_{v_{k} v^{*}} \ell_{v_{k} v^{*}}\right) \\
=r \ell_{v w_{\sigma+k}}+\ell_{k}^{\prime} \ell_{v v^{*}}-\ell_{v w_{\sigma+k}} \tilde{\ell}_{v_{k} v^{*}} \ell_{v_{k} v^{*}} / d_{v_{k}}=r \ell_{v w_{\sigma+k}},
\end{gathered}
$$

since it is easy to check that $\ell_{k}^{\prime} \ell_{v v^{*}}=\ell_{v^{*} w_{\sigma+k}}^{\prime} \ell_{v v^{*}}$ and $\ell_{v w_{\sigma+k}} \tilde{\ell}_{v_{k} v^{*}} \ell_{v_{k} v^{*}} / d_{v_{k}}$ represent the same product of weights. This completes the proof of Lemma 4.2 and hence also of Theorem 2.6 .

\section{The discriminant group and its natural represen- tation}

We consider the dual resolution graph $\Gamma$ of a good resolution of a normal surface singularity with rational homology sphere link (definitions relating to dual resolution graph, minimal good resolution, etc., are recalled in Section 12). A vertex $v$ in $\Gamma$ corresponds to an exceptional curve $E_{v}$, and an edge corresponds to an intersection of two exceptional curves. A vertex $v$ is called a 
leaf (or end) if its valency is 1 , a node if its valency is $\geq 3$. Each vertex $v$ is weighted by self-intersection number of its associated curve $E_{v}$.

Let

$$
\mathbb{E}:=\bigoplus_{v \in \operatorname{vert}(\Gamma)} \mathbb{Z} \cdot E_{v}
$$

be the lattice generated by the classes of these curves (so $\mathbb{E}$ can be identified with the homology $H_{2}(\bar{X} ; \mathbb{Z})$ of the resolution). Via the negative-definite intersection pairing $A(\Gamma)$, one has natural inclusions

$$
\mathbb{E} \subset \mathbb{E}^{\star}=\operatorname{Hom}(\mathbb{E}, \mathbb{Z}) \subset \mathbb{E} \otimes \mathbb{Q} .
$$

The discriminant group is the finite abelian group

$$
D(\Gamma):=\mathbb{E}^{\star} / \mathbb{E}
$$

whose order is $\operatorname{det}(\Gamma):=\operatorname{det}(-A(\Gamma))$. There are induced symmetric pairings of $\mathbb{E} \otimes \mathbb{Q}$ into $\mathbb{Q}$ and $D(\Gamma)$ into $\mathbb{Q} / \mathbb{Z}$.

To calculate the discriminant group, let $\left\{e_{v}\right\} \subset \mathbb{E}^{\star}$ be the dual basis of the $E_{v}$, ie,

$$
e_{v}\left(E_{v^{\prime}}\right)=\delta_{v v^{\prime}}
$$

We claim that the images of those $e_{w}$ for which $w$ is a leaf of the graph generate $D(\Gamma)$. In fact, more is true:

Proposition 5.1 Consider a collection $e_{w}$, where $w$ runs through all but one leaf of the graph $\Gamma$. Then $D(\Gamma)$ is generated by the images of these $e_{w}$.

Proof Let $E_{v}$ be any exceptional curve, with neighbors $E_{1}, \ldots, E_{r}(r \geq 1)$. Then in $\mathbb{E}^{\star}$, one verifies by dotting with any curve that

$$
E_{v}=\left(E_{v} \cdot E_{v}\right) e_{v}+\sum_{i=1}^{r} e_{i}
$$

Thus, in $D(\Gamma)$ any $e_{i}$ can be completely expressed in terms of the $e_{v}$ corresponding to one neighbor and all the other curves on the far side of that neighbor. Choose any one end curve $E_{w}$; then every curve $E_{v}$, except for the remaining end curves, has a neighbor away from $E_{w}$, and so the corresponding $e_{v}$ may be expressed in terms of outer curves. Eventually, all are expressed in terms of the remaining end curves. 
Proposition 5.2 Let $e_{1}, \ldots, e_{t}$ be the elements of the dual basis of $\mathbb{E}^{\star}$ corresponding to the $t$ leaves of $\Gamma$. Then the homomorphism $\mathbb{E}^{\star} \rightarrow \mathbb{Q}^{t}$ defined by

$$
e \mapsto\left(e \cdot e_{1}, \ldots, e \cdot e_{t}\right)
$$

induces an injection

$$
D(\Gamma)=\mathbb{E}^{\star} / \mathbb{E} \hookrightarrow(\mathbb{Q} / \mathbb{Z})^{t} .
$$

In fact, each non-trivial element of $D(\Gamma)$ gives an element of $(\mathbb{Q} / \mathbb{Z})^{t}$ with at least two non-zero entries.

Proof It suffices to show that if $e \cdot e_{i} \in \mathbb{Z}$ for $1 \leq i \leq t-1$, then $e \in \mathbb{E}$. But then the set of $e^{\prime} \in \mathbb{E}^{\star}$ for which $e \cdot e^{\prime} \in \mathbb{Z}$ is a subgroup containing $\mathbb{E}$ and these $e_{i}$, so by the last proposition must be all of $\mathbb{E}^{\star}$. Write $e=\sum r_{v} E_{v}$ as a rational combination of exceptional curves; then for every exceptional curve $E_{v}$ one has $e \cdot e_{v}=r_{v} \in \mathbb{Z}$, as desired.

It will be convenient to exponentiate, and to consider

$$
(\mathbb{Q} / \mathbb{Z})^{t} \hookrightarrow\left(\mathbb{C}^{\star}\right)^{t}
$$

via

$$
(\ldots, r, \ldots) \mapsto(\ldots, \exp (2 \pi i r), \ldots)=:[\ldots, r, \ldots] .
$$

Keeping in mind the last proposition, we summarize in the

Proposition 5.3 If the leaves of $\Gamma$ are numbered $w_{1}, \ldots, w_{t}$, then the discriminant group $D(\Gamma)$ is naturally represented by a diagonal action on $\mathbb{C}^{t}$, where the entries are $t$-tuples of $\operatorname{det}(\Gamma)$-th roots of unity. Each leaf $w_{j}$ corresponds to an element

$$
\left[e_{w_{j}} \cdot e_{w_{1}}, \ldots, e_{w_{j}} \cdot e_{w_{t}}\right]:=\left(\exp \left(2 \pi i e_{w_{j}} \cdot e_{w_{1}}\right), \ldots, \exp \left(2 \pi i e_{w_{j}} \cdot e_{w_{t}}\right)\right),
$$

and any $t-1$ of these generate $D(\Gamma)$. The representation contains no pseudoreflections, ie, non-identity elements fixing a hyperplane.

\section{Resolution graphs and the congruence condition}

Non-minimal resolutions are needed later in our inductive arguments, so we do not want to insist that $\Gamma$ corresponds to the minimal good resolution. We therefore make the following purely technical definition. 
Definition 6.1 Quasi-minimality The resolution tree $\Gamma$ is quasi-minimal if any string in $\Gamma$ either contains no $(-1)$-weighted vertex, or consists of a unique $(-1)$-weighted vertex (a string is a connected subgraph that includes no node of $\Gamma)$.

Associated to a string

$$
E=\stackrel{-b_{1}}{\stackrel{-b}{a}} \stackrel{-b_{2}}{-}---\stackrel{-b_{k}}{-}
$$

in a resolution diagram is a continued fraction

$$
n / p=b_{1}-1 / b_{2}-1 / \cdots-1 / b_{k} .
$$

The continued fraction $1 / 0$ is associated with the empty string. We will need the following standard facts about this relationship, whose proofs are left to the reader.

Lemma 6.2 Reversing a string with continued fraction $n / p$ gives one with continued fraction $n / p^{\prime}$ with $p p^{\prime} \equiv 1(\bmod n)$. Moreover, the following hold:

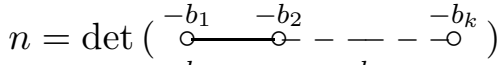

$$
\begin{aligned}
& p=\operatorname{det}\left(\stackrel{-b_{2}}{\circ}-\ldots-b^{-} b_{k}\right) \\
& p^{\prime}=\operatorname{det}\left(\stackrel{-b_{1}}{\circ}---\stackrel{-b_{k-1}}{-}\right) \text {, }
\end{aligned}
$$

and the continued fraction in the last case is $p^{\prime} / n^{\prime}$ with $n^{\prime}=\left(p p^{\prime}-1\right) / n$.

There is a unique directed quasi-minimal string for each $n / p \in[1, \infty]$, and in this case the reversed string has continued fraction $n / p^{\prime}$ with $p^{\prime}$ the unique $p^{\prime} \leq n$ with $p p^{\prime} \equiv 1(\bmod n)$.

Associate to a (not necessarily minimal) resolution graph $\Gamma$ a splice diagram $\Delta$, as in [9] (see also section 12): First, suppress all vertices of valency two in $\Gamma$, yielding a tree of the same general shape, but now with only leaves and nodes. Second, to every node $v$ and adjacent edge $e$ of $\Delta$ (or $\Gamma$ ), associate an edgeweight $d_{v e}$ as follows: removing the node from $\Gamma$, take the positive determinant $d_{v e}:=\operatorname{det}\left(\Gamma_{v e}\right)$ of the remaining connected graph $\Gamma_{v e}$ in the direction of the edge. The splice diagram has positive edge-determinants and satisfies the ideal condition (Definition 2.2 this is proved in section 12). In the unimodular case, with $\operatorname{det}(\Gamma)=1$, the weights around a node are relatively prime; but this is no longer true in general.

The discriminant group $D(\Gamma)$ acts diagonally on $\mathbb{C}^{t}$, as in Proposition 5.3 . Viewing the $z_{w}$ 's as linear functions on $\mathbb{C}^{t}, D(\Gamma)$ acts naturally on the polynomial ring $P=\mathbb{C}\left[\ldots, z_{w}, \ldots\right] ; e$ acts on monomials as

$$
\Pi z_{w}^{\alpha_{w}} \mapsto\left[-\sum\left(e \cdot e_{w}\right) \alpha_{w}\right] \Pi z_{w}^{\alpha_{w}}
$$


In other words, the group transforms this monomial by multiplying by the character

$$
e \mapsto \exp \left(-2 \pi i \sum\left(e \cdot e_{w}\right) \alpha_{w}\right)
$$

If $\Delta$ satisfies the semigroup condition, one has the notion of admissible monomials (Definition 2.4).

Definition 6.3 Congruence Condition Let $\Gamma$ be a resolution diagram, yielding a splice diagram $\Delta$ satisfying the semigroup condition. We say $\Gamma$ satisfies the congruence condition if for each node $v$, one can choose for every adjacent edge $e$ an admissible monomial $M_{v e}$ so that $D(\Gamma)$ transforms each of these monomials according to the same character.

We can write down this condition explicitly in terms of $\Gamma$ and $\Delta$.

Lemma 6.4 The matrix $\left(e_{v} \cdot e_{v^{\prime}}\right)\left(v, v^{\prime}\right.$ vertices of $\left.\Gamma\right)$ is the inverse of the matrix $A(\Gamma)=\left(E_{v} \cdot E_{v^{\prime}}\right)$.

Proof By elementary linear algebra, the matrix of the dual basis in an inner product space (such as $\mathbb{E} \otimes \mathbb{Q}$ ) is the inverse of the matrix of the original basis.

Proposition 6.5 Let $w, w^{\prime}$ be distinct leaves of $\Gamma$, corresponding to distinct leaves of $\Delta$, and let $\ell_{w w^{\prime}}$ denote their linking number. Then

$$
e_{w} \cdot e_{w^{\prime}}=-\ell_{w w^{\prime}} / \operatorname{det}(\Gamma) .
$$

Proof This is immediate by the preceding lemma and Theorem 12.2

This proposition implies that for distinct leaves $w, w^{\prime}$ the number $e_{w} \cdot e_{w^{\prime}}$ depends (except for the denominator $\operatorname{det}(\Gamma))$ only on the splice diagram $\Delta$. For a leaf $w$, the number $\left(e_{w} \cdot e_{w}\right) \operatorname{det}(\Gamma)$ is not determined solely by $\Delta$.

Proposition 6.6 Suppose we have a string from a node $v$ to an adjacent leaf $w_{1}$ in $\Gamma$ with associated continued fraction $d_{1} / p$, so $d_{1}$ is the weight at $v$ towards $w_{1}$. Let $p^{\prime}$ be the determinant of the same string with the last vertex $w_{1}$ deleted, so $p p^{\prime} \equiv 1\left(\bmod d_{1}\right)$ (see Lemma 6.2). Then (with $d_{v}$ the product of weights at $v$ )

$$
e_{w_{1}} \cdot e_{w_{1}}=-d_{v} /\left(d_{1}^{2} \operatorname{det}(\Gamma)\right)-p^{\prime} / d_{1} .
$$

(Compare this with $e_{w_{1}} \cdot e_{w_{2}}=-d_{v} /\left(d_{1} d_{2} \operatorname{det}(\Gamma)\right)$ for two leaves adjacent to v.) 
Proof Lemma 6.4, Theorem 12.2, and Lemma 12.5.

Corollary 6.7 The class of $e_{w^{\prime}}\left(w^{\prime}\right.$ a leaf) transforms the monomial $\Pi z_{w}^{\alpha_{w}}$ by multiplication by the root of unity

$$
\left[\sum_{w \neq w^{\prime}} \alpha_{w} \ell_{w w^{\prime}} / \operatorname{det}(\Gamma)-\alpha_{w^{\prime}} e_{w^{\prime}} \cdot e_{w^{\prime}}\right] .
$$

These formulas allow a direct way to check the congruence condition.

Proposition 6.8 Let $\Gamma$ be a graph whose splice diagram $\Delta$ satisfies the semigroup condition. Then the congruence condition is equivalent to the following: for every node $v$ and adjacent edge $e$, there is an admissible monomial $M_{v e}=\Pi z_{w}^{\alpha_{w}}$ ( $w$ running through the leaves in $\left.\Delta_{v e}\right)$ so that for every leaf $w^{\prime}$ of $\Delta_{v e}$,

$$
\left[\sum_{w \neq w^{\prime}} \alpha_{w} \ell_{w w^{\prime}} / \operatorname{det}(\Gamma)-\alpha_{w^{\prime}} e_{w^{\prime}} \cdot e_{w^{\prime}}\right]=\left[\ell_{v w^{\prime}} / \operatorname{det}(\Gamma)\right]
$$

Proof We first claim that if $\bar{e} \neq e$ is another edge of $v$, then $e_{w^{\prime}}$ transforms any admissible polynomial $M_{v \bar{e}}$ by the root of unity

$$
\left[\ell_{v w^{\prime}} / \operatorname{det}(\Gamma)\right] \text {. }
$$

To see this, one checks (via the definition of linking numbers) that for $\bar{w} \in \Delta_{v \bar{e}}$, one has

$$
\ell_{w^{\prime} \bar{w}}=\ell_{w^{\prime} v} \ell_{v \bar{w}}^{\prime} / d_{v \bar{e}}
$$

In particular, if

$$
d_{v \bar{e}}=\sum \beta_{\bar{w}} \ell_{v \bar{w}}^{\prime}
$$

then

$$
\sum \beta_{\bar{w}} \ell_{w^{\prime} \bar{w}}^{\prime}=\ell_{v w^{\prime}}
$$

The claim follows.

In particular, at each node $v$, checking the congruence condition on the $M_{v e}$ 's imposed by one $e_{w}$ involves only the stated equality, involving the edge in the direction of $w$.

Note that there is nothing to check for edges leading to leaves. In case $\Gamma$ is star-shaped (ie, $\Delta$ has only one node), there are no semigroup conditions, hence no congruence conditions. We will later explain these conditions completely in the two-node case. But we give one example. 
Example 6.9 9] Consider the resolution diagram

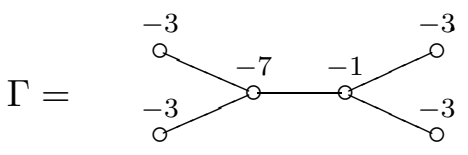

The corresponding splice diagram is

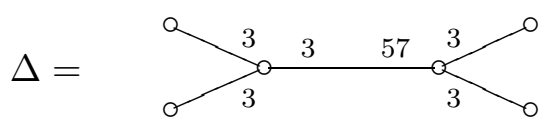

Labeling the variables $x, y, u, v$ clockwise from the bottom left leaf, one checks that an admissible monomial $u^{\alpha} v^{\beta}$ at the left node satisfies the condition of Proposition 6.8 if and only if $\alpha$ and $\beta$ are both $\equiv 2(\bmod 3)$. This is incompatible with the admissibility condition $\alpha+\beta=1$, so $\Gamma$ does not satisfy the congruence condition.

\section{Splice diagram equations with discriminant group action}

Let $\Gamma$ be a quasi-minimal resolution tree (Definition 6.1). Assume $\Gamma$ satisfies the semigroup and congruence conditions $(2.3,6.3)$. Let $\Delta$ be the corresponding splice diagram, and $z_{w}$ a variable associated to each leaf $w$. The discriminant group $D(\Gamma)$ acts on the monomials in the variables $z_{w}$.

For each node $v$, choose admissible monomials $M_{v e}$ for all the adjacent edges which transform equivariantly with respect to the action of $D(\Gamma)$. Then $D(\Gamma)$ acts on the associated equations of splice type so long as the higher order terms in these equations transform appropriately under the action of the group.

Definition 7.1 Let $\Gamma$ be a quasi-minimal resolution tree satisfying the semigroup and congruence conditions. Let $\Delta$ be the corresponding splice diagram, $z_{w}$ a variable associated to each leaf $w, M_{v e}$ an admissible monomial for each node $v$ and adjacent edge $e$ satisfying the $D(\Gamma)$-equivariance condition. Then splice diagram equations for $\Gamma$ are equations of the form

$$
\sum_{e} a_{v i e} M_{v e}+H_{v i}=0, \quad i=1, \ldots, \delta_{v}-2, \quad v \text { a node },
$$

where 
- for every $v$, all maximal minors of the matrix $\left(a_{v i e}\right)$ have full rank;

- $H_{v i}$ is a convergent power series in the $z_{w}$ 's all of whose monomials have $v$-weight $>d_{v}$;

- for each $v$, the monomials in $H_{v i}$ transform under $D(\Gamma)$ in the same way as do the $M_{v e}$ 's.

We are ready for the careful statement of the main result of this paper.

Theorem 7.2 (Splice-quotient singularities) Suppose $\Gamma$ is quasi-minimal and satisfies the semigroup and the congruence conditions. Then:

(1) Splice diagram equations for $\Gamma$ define an isolated complete intersection singularity $(X, o)$.

(2) The discriminant group $D(\Gamma)$ acts freely on a punctured neighborhood of $o$ in $X$.

(3) $Y=X / D(\Gamma)$ has an isolated normal surface singularity, and a good resolution whose associated dual graph is $\Gamma$.

(4) $X \rightarrow Y$ is the universal abelian covering.

(5) For any node $v$, the $v$-grading on functions on $Y$ (induced by the $v$ grading on $X)$ is $\operatorname{det}(\Gamma)$ times the grading by order of vanishing on the exceptional curve $E_{v}$.

(6) $X \rightarrow Y$ maps the curve $z_{w}=0$ to an irreducible curve, whose proper transform on the good resolution of $Y$ is smooth and intersects the exceptional curve transversally, along $E_{w}$. In fact the function $z_{w}^{\operatorname{det}(\Gamma)}$, which is $D(\Gamma)$-invariant and hence defined on $Y$, vanishes to order $\operatorname{det}(\Gamma)$ on this curve.

The first assertion of the theorem has already been proved in Theorem 2.6, For the second, recall (Proposition 5.3) that the fixed locus of a non-identity element of $D(\Gamma)$ is contained in some subspace $z_{w}=z_{w^{\prime}}=0$, which intersects the germ of $X$ only at the origin (Corollary [3.4). It follows that $Y$ has an isolated normal singularity, and the main point is to show the resolution dual graph equals $\Gamma$. Once that is achieved, the link of $Y$ will be a rational homology sphere whose universal abelian covering has order $\operatorname{det}(\Gamma)$, hence must be given by the abelian covering provided by the link of $X$; this gives the fourth assertion.

The fifth assertion follows immediately from the sixth if one restricts to monomials (although its general proof will involve more work). 
Lemma 7.3 Let $d=\operatorname{det}(\Gamma)$. Assuming assertion (6) of the theorem, if $z_{w}$ is the variable corresponding to a leaf then the order of vanishing of $z_{w}^{d}$ on $E_{v}$ is $\ell_{v w}\left(z_{w}^{d}\right.$ is $D(\Gamma)$-invariant, so it is defined on $\left.Y\right)$.

Proof The divisor of the function $z_{w}^{d}$ on $\bar{Y}$ has the form

$$
\left(z_{w}^{d}\right)=d D+\sum r_{u} E_{u}, \quad \text { sum over vertices } u \text { of } \Gamma \text { other than } w,
$$

Where $D$ is the proper transform of the curve given by $z_{w}^{d}=0$ in $Y$. This divisor dots to zero with each $E_{v}$, whence $r_{v}$ is the $v w$ entry of $-d A(\Gamma)^{-1}$. Theorem 12.2 then says $r_{v}=\ell_{v w}$; the $v$-weight of $z_{w}$.

By taking a closer look at the weighted blow-up as in Lemma 4.2 we will first check the theorem in the one-node case of [7] (giving a new proof for this case), and then proceed by induction on the number of nodes. This induction involves choosing an end-node $v^{*}$ of $\Gamma$ (or $\Delta$ ), and reducing to $\tilde{\Gamma}$, obtained by removing $v^{*}$ and its adjacent strings leading to leaves. But if there are no curves between $v^{*}$ and the node in the remaining direction, then we must first blow-up between these nodes, and create a new -1 curve. This will guarantee that the new $\tilde{\Gamma}$ is also quasi-minimal (and that there is a leaf corresponding to the removed $v^{*}$ ). It is easy to check the following:

Lemma 7.4 Suppose $\Gamma$ satisfies the semigroup and congruence conditions, and has adjacent nodes. Let $\Gamma^{\prime}$ be the graph obtained from blowing-up between the adjacent nodes. Then $\Gamma$ and $\Gamma^{\prime}$ have the same splice diagram and representation of the discriminant group on the space of ends. In particular, splice diagram equations for $\Gamma$ are the same for $\Gamma^{\prime}$.

We also need the quasi-minimal version of a well known and classical lemma:

Lemma 7.5 Consider a negative-definite string of rational curves

$$
\stackrel{-b_{1} \quad-b_{2}}{\longrightarrow}---b_{k}^{-}
$$

where either $b_{i}>1$ for $i=1, \ldots, k$, or $k=1$ and $b_{1}=1$. Write the continued fraction

$$
n / p=b_{1}-1 / b_{2}-1 / \cdots-1 / b_{k} .
$$

Then the cyclic quotient singularity $\mathbb{C}^{2} /\langle T\rangle$, where

$$
T(x, y)=(\exp (2 \pi i / n) x, \exp (2 \pi i p / n) y),
$$

has a resolution with the above string of exceptional curves. The proper transform of the image of $x=0$ on this resolution intersects once transversally on the right. 
Reading the string in the other direction yields $n / p^{\prime}$, where $p p^{\prime} \equiv 1(\bmod n)$. Note $n=p=p^{\prime}=1$ is allowed; in all other cases, $n>1$ and $p<n$.

\section{The case of one node}

Recall that if $\mathbb{C}^{t}$ has coordinates $z_{i}$ of weight $m_{i}$, the weighted blow-up $Z \rightarrow \mathbb{C}^{t}$ has an open covering $U_{i}$, each of which is a quotient of an affine space $V_{i}$ by a cyclic group of order $m_{i}$. $V_{1}$ has coordinates $A_{1}, \ldots, A_{t}$, related to the $z_{i}$ via

$$
z_{1}=A_{1}^{m_{1}}, z_{2}=A_{1}^{m_{2}} A_{2}, \ldots, z_{t}=A_{1}^{m_{t}} A_{t} ;
$$

$U_{1}$ equals $V_{1}$ modulo the action of the cyclic group generated by

$$
S=\left[-1 / m_{1}, m_{2} / m_{1}, \ldots, m_{t} / m_{1}\right] .
$$

A finite group $D$ of diagonal matrices acting on $\mathbb{C}^{t}$ preserves the weight filtration, so it lifts to an action on $Z$, and one has a proper birational map $Z / D \rightarrow \mathbb{C}^{t} / D$. A diagonal $\left[\beta_{1}, \ldots, \beta_{t}\right]$ acting on $\mathbb{C}^{t}$ may be lifted to one acting on $V_{1}$ via

$$
-\beta_{1} S+\left[0, \beta_{2}, \ldots, \beta_{t}\right]
$$

this lift depends on the choice of $\beta_{1}$. The naturally defined group $D_{1}$, generated by $S$ and all lifts of elements of $D$, acts on $V_{1}$, and induces an isomorphism $V_{1} / D_{1} \simeq U_{1} / D$ onto an open subset of $Z / D$. In our situation, $D_{1}$ will contain a pseudo-reflection of the form $[1 / d, 0, \ldots, 0]$. Dividing $V_{1}$ first by this action produces another affine space $\bar{V}_{1}$, with coordinates $\bar{A}_{1}, A_{2}, \ldots, A_{t}$, where $\bar{A}_{1}=$ $A_{1}^{d}$; the quotient group $\bar{D}_{1}$ acts on $\bar{V}_{1}$ with quotient $U_{1} / D$. Note that the image of the exceptional divisor is given by $\bar{A}_{1}=0$.

Consider now a quasi-minimal resolution graph $\Gamma$ with one node, given by the diagram

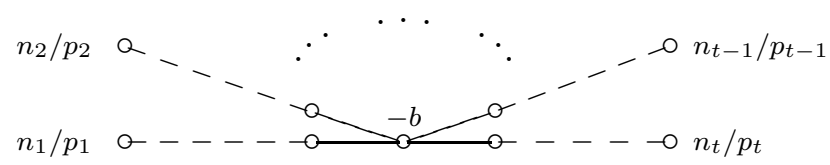

The strings of $\Gamma$ are described uniquely by the continued fractions shown, starting from the node (by the quasi-minimality condition of Definition 6.1). Set $N=n_{1} n_{2} \ldots n_{t}, N_{i}=N / n_{i}$; then $d=N\left(b-\sum_{i=1}^{t} p_{i} / n_{i}\right)=\operatorname{det}(\Gamma)$ is the determinant. The splice diagram produces leaf variables $z_{i}$ of weight $N_{i}$ for $1 \leq i \leq t$. 
The affine space $V_{1}$ above has coordinates $A_{i}, 1 \leq i \leq t$ related to the $z_{i}$ via

$$
z_{1}=A_{1}^{N_{1}}, z_{i}=A_{1}^{N_{i}} A_{i}, \quad 2 \leq i \leq t .
$$

The quotient $V_{1} \rightarrow U_{1}$ comes from dividing by

$$
S=\left[-1 / N_{1}, N_{2} / N_{1}, \ldots, N_{t} / N_{1}\right] .
$$

The discriminant group $D(\Gamma)$ acts on $\mathbb{C}^{t}$ and the weighted blow-up $Z . D(\Gamma)$ is generated by

$$
\begin{aligned}
& {\left[N_{1} /\left(n_{1} d\right)+p_{1}^{\prime} / n_{1}, N_{2} /\left(n_{1} d\right), \ldots, N_{t} /\left(n_{1} d\right)\right],} \\
& {\left[N_{1} /\left(n_{2} d\right), N_{2} /\left(n_{2} d\right)+p_{2}^{\prime} / n_{2}, \ldots, N_{t} /\left(n_{2} d\right)\right] \text {, }} \\
& \text {.. } \ldots \text {. }
\end{aligned}
$$

A simple calculation verifies the following lifts of these elements to $V_{1}$ :

$$
\begin{aligned}
& T_{1}=\left[1 /\left(n_{1} d\right)+p_{1}^{\prime} / N, \quad-p_{1}^{\prime} / n_{2}, \ldots,-p_{1}^{\prime} / n_{t}\right] \\
& T_{2}=\left[1 /\left(n_{2} d\right), p_{2}^{\prime} / n_{2}, 0, \ldots, 0\right] \\
& \text {... } \\
& T_{t}=\left[1 /\left(n_{t} d\right), 0, \ldots, 0, p_{t}^{\prime} / n_{t}\right] .
\end{aligned}
$$

Consider the lifted group $D_{1}$ generated by $S$ and these $T_{i}$. Writing $p_{1} p_{1}^{\prime}=$ $k n_{1}+1$, we may replace $S$ and $T_{1}$ by

$$
\begin{aligned}
& \bar{S}=S^{p_{1}^{\prime}} T_{1}^{n_{1}}=[1 / d, 0, \ldots, 0] \\
& \bar{T}=S^{k} T_{1}^{p_{1}}=\left[1 / N+p_{1} /\left(n_{1} d\right),-1 / n_{2}, \ldots,-1 / n_{t}\right],
\end{aligned}
$$

and then replace $\bar{T}$ by

$$
\tilde{T}=\bar{T} T_{2}^{p_{2}} \ldots T_{t}^{p_{t}}=\left[1 / N+\sum_{i=1}^{t} p_{i} /\left(n_{i} d\right), 0, \ldots, 0\right]=[b / d, 0, \ldots, 0] .
$$

Since $\tilde{T}$ is a power of $\bar{S}, D_{1}$ is generated by the pseudoreflection $\bar{S}$ and the $T_{i}, i>1$. Dividing $V_{1}$ by $\bar{S}$ gives the affine space $\bar{V}_{1}$, with coordinates $\bar{A}_{1}=$ $A_{1}^{d}, A_{2}, \ldots, A_{t}$, and group action generated by

$$
\begin{aligned}
& \bar{T}_{2}=\left[1 / n_{2}, p_{2}^{\prime} / n_{2}, 0, \ldots, 0\right] \\
& \bar{T}_{t}=\left[1 / n_{t}, 0, \ldots, 0, p_{t}^{\prime} / n_{t}\right] .
\end{aligned}
$$

In this one-node case, the admissible monomials are $z_{i}^{n_{i}}, i=1, \ldots, t$, and the splice diagram equations are of the form

$$
\sum_{j=1}^{t} a_{i j} z_{j}^{n_{j}}+H_{i}(\underline{z})=0, i=1, \ldots, t-2 .
$$


A monomial $\prod z_{j}^{\alpha_{j}}$ is allowed to appear in one of the convergent power series $H_{i}$ iff it transforms under $D(\Gamma)$ as do the admissible monomials, and if the $v$-weight $\sum \alpha_{j} N_{j}$ is $>N$. By the assumption on the coefficient matrix $\left(a_{i j}\right)$, one may take linear combinations of the series and rewrite as

$$
z_{i}^{n_{i}}+a_{i} z_{t-1}^{n_{t-1}}+b_{i} z_{t}^{n_{t}}+H_{i}(\underline{z})=0, \quad 1 \leq i \leq t-2
$$

with $a_{i} b_{j}-a_{j} b_{i} \neq 0$ for all $i \neq j$, and all $a_{i}$ and $b_{i}$ nonzero. The associated graded ring with respect to the weight filtration is the familiar Brieskorn complete intersection (except that some $n_{i}=1$ is possible).

As in Section 4 , one finds the proper transform $X_{1}$ on $V_{1}$ defined by:

$$
\begin{aligned}
1+a_{1} A_{t-1}^{n_{t-1}}+b_{1} A_{t}^{n_{t}}+A_{1} J_{1}(\underline{A}) & =0 \\
A_{i}^{n_{i}}+a_{i} A_{t-1}^{n_{t-1}}+b_{i} A_{t}^{n_{t}}+A_{1} J_{i}(\underline{A}) & =0, \quad 2 \leq i \leq t-2 .
\end{aligned}
$$

Here one has used the weight condition on the $H_{i}$ to define

$$
H_{i}\left(A_{1}^{N_{1}}, A_{1}^{N_{2}} A_{2}, \ldots, A_{1}^{N_{t}} A_{t}\right)=A_{1}^{N+1} J_{i}(\underline{A}) .
$$

Intersecting with the exceptional divisor $A_{1}=0$ gives the polynomials equations

$$
\begin{aligned}
1+a_{1} A_{t-1}^{n_{t-1}}+b_{1} A_{t}^{n_{t}} & =0, \\
A_{i}^{n_{i}}+a_{i} A_{t-1}^{n_{t-1}}+b_{i} A_{t}^{n_{t}} & =0, \quad 2 \leq i \leq t-2 .
\end{aligned}
$$

This is a smooth curve, so $X_{1}$ is smooth. Since $\bar{S}$ leaves $A_{2}, \ldots, A_{t}$ invariant and acts equivariantly on the equations, it follows that each term $A_{1} J_{i}\left(A_{k}\right)$ must be invariant, and hence is a power series $\bar{A}_{1} \bar{J}_{i}$ in $\bar{A}_{1}, A_{2}, \ldots, A_{t}$. So the quotient $\bar{X}_{1}$ of $X_{1}$ by $\bar{S}$ is defined on $\bar{V}_{1}$ via

$$
\begin{aligned}
1+a_{1} A_{t-1}^{n_{t-1}}+b_{1} A_{t}^{n_{t}}+\bar{A}_{1} \bar{J}_{1} & =0, \\
A_{i}^{n_{i}}+a_{i} A_{t-1}^{n_{t-1}}+b_{i} A_{t}^{n_{t}}+\bar{A}_{1} \bar{J}_{i} & =0, \quad 2 \leq i \leq t-2 .
\end{aligned}
$$

We divide $\bar{X}_{1}$ by the action of $\bar{D}_{1}:=D_{1} / \bar{S}$, which is generated by the images of $\bar{T}_{i}, i=2, \ldots, t$. First, the group acts transitively on the connected components of $\bar{A}_{1}=0$, since, eg, every component contains a point with $A_{t}=0$ (cf. Lemma 4.1); since $A_{1}=0$ in $X_{1}$ is smooth, the image of the exceptional divisor $\bar{A}_{1}=0$ is irreducible. Next, the action is free off $\bar{A}_{1}=0$, and fixed points occur exactly when another coordinate is 0 . At a point of $\bar{X}_{1}$ where $\bar{A}_{1}=A_{2}=0$, the above equations (9) determine $A_{k}^{n_{k}}$ for $k=3, \ldots, t$ uniquely. Thus there are $n_{3} \ldots n_{t}$ such points, and they are permuted by the subgroup generated by $\bar{T}_{3}, \ldots, \bar{T}_{t}$. So, there is one orbit of such fixed points, and the stabilizer is generated by $\bar{T}_{2}$. The equations imply that $\bar{A}_{1}, A_{2}$ are local coordinates at such a point.

We have now a familiar local picture as described in Lemma 7.5 and following comments: divide $\mathbb{C}^{2}$ by the action $\left[1 / n_{2}, p_{2}^{\prime} / n_{2}\right]$, resolve the cyclic quotient 
singularity according to the corresponding string in $\Gamma$ (if $n_{2}=1$ we blow-up once), and consider the proper transforms of the images of the two coordinates axes on $\mathbb{C}^{2}$. We get a string of curves

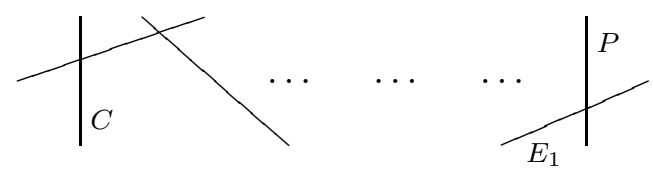

The left-hand curve $C$ corresponds to the transform of $\bar{A}_{1}=0$ (which will thus be a central exceptional curve in a resolution of $X$ ), the right curve $P$ is the proper transform of $A_{2}=0$, and the remaining curves form the string of exceptional curves that resolve the cyclic quotient singularity. The continued fraction expansion from left to right is $n_{2} / p_{2} . A_{2}^{n_{2}}$ vanishes $n_{2}$ times along $P$ and $p_{2}^{\prime}$ times along the adjacent exceptional curve $E_{1}$; and, $\bar{A}_{1}^{n_{2}}$ vanishes once along $E_{1}$.

Putting this all together for the other $U_{i}$ and $V_{i}$, we conclude that the quotient variety $X / D(\Gamma)$ has a resolution consisting of a smooth central curve, and $t$ rational strings emanating from it corresponding to $n_{i} / p_{i}, 1 \leq i \leq t$. It remains to show the central curve is rational, and its self-intersection is $-b$ (equivalently, the determinant $\bar{d}$ of the intersection pairing equals $d$ ).

Restricting to the exceptional divisor $\bar{A}_{1}=0$ in $\bar{X}_{1} / \bar{D}_{1}=X_{1} / D_{1}$ gives new variables $C_{i}=A_{i}^{n_{i}}, 2 \leq i \leq t$, in which the defining equations in (8) become linear. So, the quotient is a line in the coordinate space, hence is rational.

Finally, note that $z_{2}^{d n_{2}}$ is invariant under the discriminant group, hence is a function on $X / D(\Gamma)$; we consider its proper transform in the minimal resolution of $\bar{V}_{1} / \bar{D}_{1}$. (The proper transform misses $V_{2}$, so is completely contained in $V_{1}$.) Note first that

$$
z_{2}^{d n_{2}}=A_{1}^{d N} A_{2}^{d n_{2}}=\bar{A}_{1}^{N} A_{2}^{d n_{2}} .
$$

By an earlier remark, $z_{2}^{d n_{2}}$ vanishes $N / n_{2}+d p_{2}^{\prime}$ times along $E_{1}$, and $d n_{2}$ times along $P$. Thus the divisor of the function $z_{2}^{d n_{2}}$ has the form

$$
\left(z_{2}^{d n_{2}}\right)=d n_{2} P+\left(N / n_{2}+d p_{2}^{\prime}\right) E_{1}+\sum_{i>1} r_{i} E_{i}
$$

the last sum being over all the other exceptional curves in the quasi-minimal resolution. Since the divisor of a function dots to zero with each $E_{i}$, we see that

$$
-\frac{1}{d n_{2}}\left(\left(N / n_{2}+d p_{2}^{\prime}\right) E_{1}+\sum_{i>1} r_{i} E_{i}\right)
$$


represents the element $e_{1}$ in the dual $\mathbb{E}^{*}$ of $\mathbb{E}=\bigoplus \mathbb{Z} \cdot E_{v}$. On the other hand, $e_{1}=\sum_{i}\left(e_{1} \cdot e_{i}\right) E_{i}$, so

$$
e_{1} \cdot e_{1}=-\left(N / n_{2}+d p_{2}^{\prime}\right) /\left(d n_{2}\right) .
$$

Comparing this with the value $e_{1} \cdot e_{1}=-N /\left(n_{2}^{2} \bar{d}\right)-p_{2}^{\prime} / n_{2}$ of Proposition 6.6 yields that $\bar{d}=d$, as desired.

So, the constructed singularity has a resolution with dual graph our original $\Gamma$. Since we have constructed an abelian covering of degree $d$ equal to the discriminant of our singularity, the map $X \rightarrow X / D(\Gamma)$ must be the universal abelian covering.

To prove the assertion (6) of the main theorem, note that the proper transform of $z_{i}^{n_{i} d}$ on the given quasi-minimal resolution intersects the $i$-th quotient string, once transversally on the end, with multiplicity $n_{i} d$.

Finally, for assertion (5) of the main theorem, the central exceptional curve in the resolution of $X$ maps to the central curve $E_{v}$ in the resolution of $Y$, so the gradings on functions on $Y$ (ie, $D(\Gamma)$-invariant functions on $X$ ) given by order of vanishing on these curves agree up to a constant multiple. Since the central curve of the resolution of $X$ is the curve obtained by blowing up the $v$-grading, order of vanishing on it is given by the $v$-grading. This proves (5) up to a multiple; that the multiple is correct is confirmed in Lemma 7.3

\section{The inductive procedure}

Assume we have a set of splice diagram equations for a quasi-minimal $\Gamma$, which is assumed to have more than one node. The inductive assumption is that the theorem is true for a graph with fewer nodes. As mentioned, we may assume (after blowing-up) that any two nodes of $\Gamma$ have at least one curve between them. Let $\Delta$ be the splice diagram and $v^{*}$ an end-node, viewed on the left side of the diagrams:

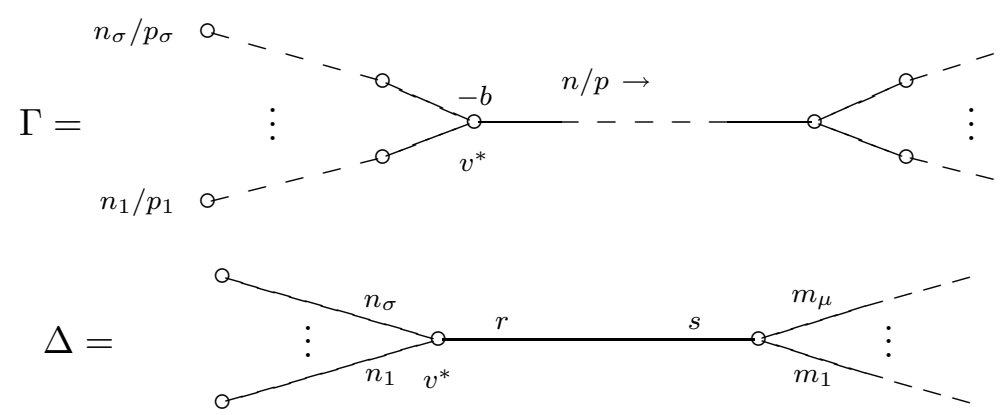


The continued fractions from $v^{*}$ to the leaves are given by $n_{i} / p_{i}, 1 \leq i \leq \sigma$, and from $v^{*}$ to the adjacent node by $n / p$. Let $N=n_{1} \ldots n_{\sigma}, N_{i}=N / n_{i}, M=$ $m_{1} \ldots m_{\mu}$. One has the determinant calculation

$$
s=N n\left(b-\sum_{i=1}^{\sigma} p_{i} / n_{i}-p / n\right) .
$$

Moreover, if $d=\operatorname{det}(\Gamma)=\operatorname{det}(-A(\Gamma))$, we have the relation (see Proposition 12.3)

$$
r s-M N=d n .
$$

Induction will involve $\tilde{\Gamma}$, obtained by removing from $\Gamma$ the vertex corresponding to $v^{*}$ plus the $\sigma$ strings of rational curves to the leaves. The corresponding splice diagram $\tilde{\Delta}$ has a new leaf $w^{*}$ in the location of $v^{*}$, but one loses the $\sigma$ leaves of $\Delta$ adjacent to $v^{*}$. The left sides of $\tilde{\Gamma}$ and $\tilde{\Delta}$ are

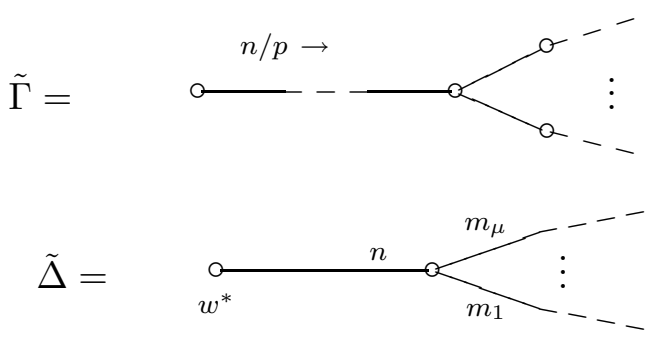

For edges of $\tilde{\Delta}$ which point away from $w^{*}$, the weights are the same as they were for $\Delta$. For the edge pointing toward $w^{*}$ at a node $v$ the new edge weight is now given by (see Lemma 12.7):

$$
\tilde{d}_{v 1}=\frac{1}{d}\left(r d_{v 1}-N\left(d_{v} / d_{v 1}\right)\left(\ell_{w^{*} v}^{\prime}\right)^{2}\right) .
$$

Note also that

$$
r=\operatorname{det}(\tilde{\Gamma})=\operatorname{det}(-A(\Gamma)) .
$$

We use exactly the same notation as in Section 4 for the variables and equations for the singularity $X(\Delta)$, including the $v^{*}$-blow-up to $X_{1} \subset V_{1}$. The coordinates corresponding to the left leaves are $x_{1}, \ldots, x_{\sigma}$ and those corresponding to the right leaves are $y_{1}, \ldots, y_{\tau}$. The coordinate space $V_{1}$ has coordinates $A_{i}, B_{j}$, related to the $x_{i}, y_{j}$ by

$$
x_{1}=A_{1}^{N_{1} r} ; x_{i}=A_{1}^{N_{i} r} A_{i}, \quad i=2, \ldots, \sigma ; \quad y_{j}=A_{1}^{N \ell_{j}^{\prime}} B_{j}, \quad j=1, \ldots, \tau,
$$


where we are abbreviating $\ell_{j}^{\prime}:=\ell_{v^{*} w_{\sigma+j}}^{\prime}$ (the reduced linking number of $v^{*}$ and one of the $\tau$ outer leaves; similarly, we will write $\ell_{j k}$ for the linking numbers between two such leaves). On $V_{1}$, the cyclic group action is generated by

$$
S=\left[-1 / r N_{1}, n_{1} / n_{2}, \ldots, n_{1} / n_{\sigma} ; n_{1} \ell_{1}^{\prime} / r, \ldots\right] .
$$

We lift the discriminant group $D(\Gamma)$ to a group $D_{1}$ (of order $d N_{1} r$ ) acting on $V_{1}$ and, by equivariance of the action, on $X_{1}$.

The leaf-generators of $D(\Gamma)$ acting in $x_{i}, y_{j}$ coordinates are written as follows, where a semi-colon distinguishes the first $\sigma$ entries from the last $\tau$ :

$$
\begin{aligned}
& {\left[r N_{1} /\left(n_{1} d\right)+p_{1}^{\prime} / n_{1}, r N_{2} /\left(n_{1} d\right), \ldots, r N_{\sigma} /\left(n_{1} d\right) ; \ell_{1}^{\prime} N_{1} / d, \ldots\right]} \\
& {\left[r N_{1} /\left(n_{2} d\right), r N_{2} /\left(n_{2} d\right)+p_{2}^{\prime} / n_{2}, \ldots, r N_{\sigma} /\left(n_{2} d\right) ; \ell_{1}^{\prime} N_{2} / d, \ldots\right]} \\
& \ldots, \ldots \\
& {\left[r N_{1} /\left(n_{\sigma} d\right), r N_{2} /\left(n_{\sigma} d\right), \ldots, r N_{\sigma} /\left(n_{\sigma} d\right)+p_{\sigma}^{\prime} / n_{\sigma} ; \ell_{1}^{\prime} N_{\sigma} / d, \ldots\right]} \\
& {\left[\ell_{1}^{\prime} N_{1} / d, \ldots, \ell_{1}^{\prime} N_{\sigma} / d ; \ell_{11} / d, \ell_{12} / d, \ldots\right]} \\
& \quad \ldots \quad \ldots \\
& {\left[\ell_{\tau}^{\prime} N_{1} / d, \ldots, \ell_{\tau}^{\prime} N_{\sigma} / d ; \ell_{\tau 1} / d, \ell_{\tau 2} / d, \ldots\right]}
\end{aligned}
$$

One verifies by substituting from (15) that the following give lifts of these generators of $D(\Gamma)$ to the coordinates of $V_{1}$ :

$$
\begin{aligned}
& T_{1}=\left[1 /\left(n_{1} d\right)+p_{1}^{\prime} /(r N),-p_{1}^{\prime} / n_{2}, \ldots, \quad-p_{1}^{\prime} / n_{\sigma} ;-p_{1}^{\prime} \ell_{1}^{\prime} / r, \ldots\right] \\
& T_{2}=\left[1 /\left(n_{2} d\right), p_{2}^{\prime} / n_{2}, 0, \ldots, 0 ; 0, \ldots\right] \\
& \text {... ... } \\
& T_{\sigma}=\left[1 /\left(n_{\sigma} d\right), 0, \ldots, 0, p_{\sigma}^{\prime} / n_{\sigma} ; 0, \ldots\right] \\
& R_{1}=\left[\ell_{1}^{\prime} /(r d), 0, \ldots, 0 ; \ell_{11} / d-N\left(\ell_{1}^{\prime}\right)^{2} /(r d), \ell_{12} / d-N \ell_{1}^{\prime} \ell_{2}^{\prime} /(r d), \ldots\right] \\
& R_{\tau}=\left[\ell_{\tau}^{\prime} /(r d), 0, \ldots, 0 ; \ell_{\tau 1} / d-N \ell_{\tau}^{\prime} \ell_{1}^{\prime} /(r d), \ell_{\tau 2} / d-N \ell_{\tau}^{\prime} \ell_{2}^{\prime} /(r d), \ldots\right]
\end{aligned}
$$

The lifted discriminant group $D_{1}$ is generated by $S$ and the $T_{i}$ and $R_{j}$. Writing $p_{1} p_{1}^{\prime}=k n_{1}+1$, replace the generators $S$ and $T_{1}$ by new generators

$$
\begin{aligned}
& S^{\prime}=S^{p_{1}^{\prime}} T_{1}^{n_{1}}=[1 / d, 0, \ldots, 0 ; 0, \ldots] \\
& T^{\prime}=S^{k} T_{1}^{p_{1}}=\left[1 /(r N)+p_{1} /\left(n_{1} d\right),-1 / n_{2}, \ldots,-1 / n_{\sigma} ; \quad-\ell_{1}^{\prime} / r, \ldots\right] .
\end{aligned}
$$

Also replace $T^{\prime}$ by $\tilde{T}$, where

$$
\tilde{T}^{-1}=T^{\prime} T_{2}^{p_{2}} \ldots T_{\sigma}^{p_{\sigma}}=\left[1 /(r N)+\sum_{i=1}^{\sigma} p_{i} /\left(d n_{i}\right), 0, \ldots, 0 ;-\ell_{1}^{\prime} / r, \ldots\right] .
$$


Recall (Lemma 4.1) that $X_{1}$ is smooth save for the $N_{1}$ points on the exceptional curve $A_{1}=0$ where all $B_{k}$ are 0 ; note that the group generated by $T_{2}, \ldots, T_{\sigma}$ acts transitively on them, so dividing by the action will give a connected exceptional curve. $D_{1}$ also acts on $X_{1}$.

Divide $V_{1}$ by the pseudo-reflection $S^{\prime}$, giving a degree $d$ covering $V_{1} \rightarrow \bar{V}_{1}$, which is unramified off the divisor $A_{1}=0$. In the new affine space $\bar{V}_{1}$, we have coordinates $\bar{A}_{1}=A_{1}^{d}, A_{2}, \ldots, A_{\sigma}, B_{1}, \ldots, B_{\tau}$.

The action of the quotient $\bar{D}_{1}=D_{1} /\left\langle S^{\prime}\right\rangle$ on $\bar{V}_{1}$ is generated by multiplying the first entries of the old generators by $d$, yielding new generators

$$
\begin{aligned}
& \bar{T}=\left[-d /(r N)-\sum_{i=1}^{\sigma} p_{i} / n_{i}, 0, \ldots, 0 ; \ell_{1}^{\prime} / r, \ldots\right] \\
& \bar{T}_{2}=\left[1 / n_{2}, p_{2}^{\prime} / n_{2}, 0, \ldots, 0 ; 0, \ldots\right] \\
& \text {... ... } \\
& \bar{T}_{\sigma}=\left[1 / n_{\sigma}, 0, \ldots, p_{\sigma}^{\prime} / n_{\sigma} ; 0, \ldots\right] \\
& \bar{R}_{1}=\left[\ell_{1}^{\prime} / r, 0, \ldots, 0 ; \ell_{11} / d-N\left(\ell_{1}^{\prime}\right)^{2} /(r d), \ell_{12} / d-N \ell_{1}^{\prime} \ell_{2}^{\prime} /(r d), \ldots\right]
\end{aligned}
$$

Equations (11) and (12) imply

$$
-d /(r N)-\sum_{i=1}^{\sigma} p_{i} / n_{i}=M /(r n)+p / n-b,
$$

whence the generator $\bar{T}$ above may be rewritten as

$$
\bar{T}=\left[M /(r n)+p / n, 0, \ldots, 0 ; \ell_{1}^{\prime} / r, \ell_{2}^{\prime} / r, \ldots\right] .
$$

The quotient $\bar{X}_{1}$ of $X_{1}$ by $S^{\prime}$ is defined by the same equations as $X_{1}$ (see equations (3)), except that $A_{1}^{d}$ is replaced by $\bar{A}_{1} \cdot \bar{X}_{1}$ and the exceptional divisor are smooth except at the $N_{1}$ points with all $B_{k}=0$, and they form one $\bar{D}_{1}$-orbit. As in Section 4, at such a point, the coordinates $A_{i}$ for $i>1$ are non-zero, and can be solved locally as convergent power series in $\overline{A_{1}}, B_{1}, \ldots, B_{\tau}$ with non-zero constant term. Thus, around each one of these singular points $\bar{X}_{1}$ is described by equations in those variables.

Lemma 9.1 These equations are splice diagram equations for $\tilde{\Delta}$.

Proof The proof is essentially the same as Lemma 4.2. we verify that the equations have appropriate admissible monomials at every node, and all the other terms have higher order. The edge-weights of $\tilde{\Delta}$ are the same as those 
for $\Delta$ in directions away from $v^{*}$, while at a node $v$, the new edge weight $\tilde{d}_{v i}$ is $1 / d$ times the value it had in Section 4 (compare equations (4) and (13)). Monomials involve powers of $\bar{A}_{1}$ rather than $A_{1}$, so the " $Q$ " term in the proof of Lemma 4.2 is also divided by $d$. The right side of equation (7) is therefore now also divided by $d$; otherwise the proof goes through almost verbatim.

Still considering one such singular point of $\bar{X}_{1}$, the stabilizer is the subgroup $G$ of $\bar{D}_{1}$ generated by $\bar{T}, \bar{R}_{1}, \ldots, \bar{R}_{\tau}$. This group acts effectively on the space whose coordinates are $\bar{A}_{1}, B_{1}, \ldots, B_{\tau}$. Rewrite the group generators as $\tau+1-$ tuples:

$$
\begin{aligned}
& {\left[M /(r n)+p / n, \ell_{1}^{\prime} / r, \ell_{2}^{\prime} / r, \ldots\right],} \\
& {\left[\ell_{i}^{\prime} / r, \ell_{i 1} / d-N \ell_{1}^{\prime} \ell_{i}^{\prime} /(r d), \ell_{i 2} / d-N \ell_{2}^{\prime} \ell_{i}^{\prime} /(r d), \ldots\right], \quad i=1, \ldots, \tau .}
\end{aligned}
$$

Lemma 9.2 The $\tau+1$ generators above are exactly the leaf generators for the discriminant group associated to the resolution diagram $\tilde{\Gamma}$. In particular, $G$ maps isomorphically onto $D(\tilde{\Gamma})$, viewed as a diagonal subgroup of $\left(\mathbb{C}^{*}\right)^{\tau+1}$.

Proof The only "new" weights on edges of $\tilde{\Delta}$ are those pointing towards $w^{*}$; other edge-weights are the same for $\Delta$ and $\tilde{\Delta}$. Recall that $r=\operatorname{det}(\tilde{\Gamma})$. By Proposition [6.6] and Corollary 6.7, the first generator above is exactly the leaf generator of $D(\tilde{\Gamma})$ corresponding to $w^{*}$. Similarly, the first entries of the remaining $\tau$ generators above are exactly those of the corresponding leaf generators for $D(\tilde{\Gamma})$.

So, we need to compute the last $\tau$ entries for the last $\tau$ generators. Denote the linking numbers for $\tilde{\Delta}$ between pairs of these as $\tilde{\ell}_{i j}$ (keeping in mind the special definition when $i=j$ as in Proposition (6.6). To prove the lemma, we must show that for all $i$ and $j$

$$
\tilde{\ell}_{i j} / r=\ell_{i j} / d-N \ell_{i}^{\prime} \ell_{j}^{\prime} / r d
$$

When $i=j$ this equation is just a special case of Lemma 12.7 so assume $i \neq j$. Denote by $v$ the vertex where the paths from $w^{*}$ to $w_{i}$ and $w_{j}$ diverge. Then

$$
\tilde{\ell}_{i j}=\frac{\ell_{i j}}{d_{v 1}} \tilde{d}_{v 1}=\frac{\ell_{i j}}{d d_{v 1}}\left(r d_{v 1}-N\left(d_{v} / d_{v 1}\right)\left(\ell_{w^{*} v}^{\prime}\right)^{2}\right) .
$$

Substituting this in the equation to be proved reduces it to the equation $\ell_{i}^{\prime} \ell_{j}^{\prime}=$ $\frac{\ell_{i j}}{d_{v 1}}\left(d_{v} / d_{v 1}\right)\left(\ell_{w^{*} v}^{\prime}\right)^{2}$. This is an easily checked equation between products of splice diagram weights. 
The last two lemmas together imply that $Y_{1}:=\bar{X}_{1} / \bar{D}_{1}$ has a singular point which is a quotient of splice diagram equations for $\tilde{\Delta}$ by $D(\tilde{\Gamma})$. By induction, this singularity has a resolution dual graph $\tilde{\Gamma}$, and one knows where the proper transform of the exceptional fiber intersects the diagram (ie, in the location of $\left.w^{*}\right)$. The only other singular points of $Y_{1}$ come from fixed points of the group action.

Lemma 9.3 $\bar{D}_{1}$ acts transitively on the points of $\bar{X}_{1}$ with $\bar{A}_{1}=A_{2}=0$. At such a point, $\bar{A}_{1}$ and $A_{2}$ are local analytic coordinates, the stabilizer is generated by $\bar{T}_{2}$, and the image of the point on $Y_{1}$ is an $n_{2} / p_{2}$ cyclic quotient singularity.

Proof As before, the equations (3) (in which now $A_{1}$ only occurs to $d$-th powers which have been replaced by $\bar{A}_{1}$ ) show that no other coordinates can be 0 , and that $\bar{A}_{1}$ and $A_{2}$ are local coordinates (see also Lemma 4.1).

Recall that the order of $\bar{D}_{1}$ is $d r N_{1} / d=r N_{1}$ and that the order of $G$ is $\operatorname{det}(-A(\tilde{\Gamma}))=r$. The subgroup $G$ of $\bar{D}_{1}$, viewed as acting on the coordinates $\bar{A}_{1}, B_{1}, \ldots, B_{\tau}$, contains no pseudoreflections (it is a discriminant group, so apply Proposition [5.3); therefore, no element of $G$ stabilizes the point in question. Further, the subgroup generated by $\bar{T}_{3}, \ldots, \bar{T}_{\sigma}$, which has order $n_{3} \ldots n_{\sigma}=N / n_{1} n_{2}$, acts on the points in question but only changing their entries in the slots $A_{3}, \ldots, A_{\sigma}$. In particular, $G$ and the $\bar{T}_{i}$ for $i>2$ generate a subgroup of index $n_{2}$ in $\bar{D}_{1}$ which acts freely on the points, and the stabilizer of the point is generated by $\bar{T}_{2}$. Thus there are $r n_{3} \ldots n_{\sigma}$ points in the orbit, and their image is an $n_{2} / p_{2}$ cyclic quotient singularity. Finally, to see that $\bar{D}_{1}$ acts transitively on the points in question, we must show there are $r n_{3} \ldots n_{\sigma}$ points with $\overline{A_{1}}=A_{2}=0$; but this is given in Lemma 4.1

Now, the exceptional divisor of $Y_{1}$ is connected, and the last two lemmas show it is analytically irreducible at the singular points; thus, the exceptional divisor is itself irreducible. Take the resolution of the above $n_{2} / p_{2}$ cyclic quotient singularity on $Y_{1}$ (if $n_{2}=1$, this means blow-up a smooth point); then, as in section 8 . we get a string of rational curves starting from the curve $\bar{A}_{1}=0$ with continued fraction $n_{2} / p_{2}$, and the proper transform of the curve $A_{2}=0$ is smooth and intersects transversally in one point the end curve of this string. Since the curve $A_{2}=0$ arises from the curve $x_{2}=0$, this agrees with the last part (6) of Theorem 7.2 .

The action of $\bar{D}_{1}$ on the curve $\bar{A}_{1}=0$ reduces to the direct product of $G$ acting in the $B$ coordinates and the group generated by $\bar{T}_{2}, \ldots, \bar{T}_{\sigma}$ acting in the $A$ 
coordinates. It thus acts freely except on the orbit of $\tilde{\Delta}$ splice diagram singularities (which occur at the points where all the $B_{k}$ are zero), and except on points where some $A_{i}=0(i>1)$. The latter lead to cyclic quotient singularities on dividing by $\bar{D}_{1}$. The quotient $Y_{1}=\bar{X}_{1} / \bar{D}_{1}$ thus has one splice-quotient singularity and, for each $i=2, \ldots, \sigma$, one $n_{i} / p_{i}$ cyclic quotient singularity, and is otherwise smooth along its (irreducible) exceptional divisor. We have already verified that the image of $x_{i}=0$ (for $i>1$ ) on $Y$ has proper transform on $Y_{1}$ vanishing correctly on the desired end-curves.

One can see the rest of the $v^{*}$ blow-up of $X$ by inverting $x_{2}$, since the origin is an isolated point in the locus $x_{1}=x_{2}=0$ (Corollary 3.4). The resulting space $Y_{2}$ adds an $n_{1} / p_{1}$ cyclic quotient (but misses the $n_{2} / p_{2}$ one). Denote by $\bar{Y}$ the union of $Y_{1}$ and $Y_{2}$. There is a partial resolution $\bar{Y} \rightarrow X / D(\Gamma)$ whose exceptional curve is irreducible, and along which sit (in a known way) $\sigma$ cyclic quotient singularities and a splice-quotient corresponding to $\tilde{\Gamma}$. Taking the resolutions of all the quotient singularities as well as the splice-quotient (which by the induction assumption has resolution graph $\tilde{\Gamma}$ ) gives a resolution $\tilde{Y} \rightarrow \bar{Y} \rightarrow X / D(\Gamma)$, with resolution dual graph almost guaranteed to be exactly $\Gamma$. The only point to check is that the proper transform of the exceptional curve of $\bar{Y}$ in $\tilde{Y}$ is a smooth rational curve of the correct self-intersection. But that is achieved simply by repeating the entire procedure at a different end-node $v^{\prime}$ of $\Gamma$; the curve in question is then seen as a part of the resolution dual graph of type $\Gamma^{\prime}$ ( $\Gamma$ minus $v^{\prime}$ and its adjacent strings), hence has the desired properties.

The assertion about the proper transform on $\bar{Y}$ of the image of $y_{j}=0$ follows by induction, by considering the role of $B_{j}=0$ for the splice-quotient corresponding to $\tilde{\Gamma}$.

We have proved all but part (5) of Theorem [7.2. For the node $v^{*}$ that we just blew up, this part follows by the same argument as in Section 8 for the one-node case. For any other node $v$, we proceed by induction, comparing the factorizations $X_{1} \rightarrow X \rightarrow Y$ and $X_{1} \rightarrow \bar{X}_{1} \rightarrow Y_{1} \rightarrow Y$, and the relevant valuations and weightings at various points. Recall (Lemma 4.2) that $X_{1}$ has $N_{1}$ splice-type singularities for the splice diagram $\Delta^{\prime}$, obtained by deleting from $\Delta$ the node $v^{*}$ and its outer leaves, replacing by a new leaf $w^{*}$, and adjusting weights as in equation (44). (We had previously denoted this diagram by $\tilde{\Delta}$; but in the current proof, that notation is already being used, and means that the weights in (44) are divided by $d$, as in equation (13).)

Start with a function $g$ on $Y$ which vanishes to order $k$ along $E_{v}$. Viewed on the partial resolution $Y_{1}, g$ vanishes to the same order in the resolution of the singular point, which is a splice-quotient for $\tilde{\Gamma}$. Lifting further via $\bar{X}_{1} \rightarrow Y_{1}$, 
by induction $g$ is in the $r k$ th piece of the filtration (corresponding to the node $v$ in the splice diagram $\tilde{\Delta}$ ) at each of the $N_{1}$ splice-type singularities; we use $r=\operatorname{det}(\tilde{\Gamma})$. Finally, pulling back leaf variables under the pseudo-reflection quotient map $X_{1} \rightarrow \bar{X}_{1}$ multiplies $v$-weights by $d$, via comparison of $\tilde{\Delta}$ and $\Delta^{\prime}$; since the map on the associated gradeds of corresponding singular points is easily seen to be injective (simply replace $\bar{A}$ by $A_{1}^{d}$ ), the pullback of $g$ to $X_{1}$ now has weight $d r k$ in each of the $N_{1}$ associated gradeds.

We next look at pulling back a function $h$ from $X$ to $X_{1}$, and show that the induced map from the $v$-associated graded of $X$ to the direct sum of the $N_{1}$ associated gradeds on $X_{1}$ multiplies by degree $r$ and is injective. Once this claim is established, we see that the pull-back of our original $g$ to $X$ must have $v$-filtration weight equal to $d k$, in order to get the correct weight at each point of $X_{1}$. The following lemma thus completes part (5) of the theorem for these nodes.

Lemma 9.4 The map $X_{1} \rightarrow X$ induces a natural map from the $v$-associated graded of $X$ to the direct sum of $N_{1}$ associated gradeds for the splice singularities on $X_{1}$. This map is injective and multiplies degrees by $r$.

Proof The map on polynomial rings

$$
R=\mathbb{C}\left[x_{i}, y_{j}\right] \rightarrow R_{1}=\mathbb{C}\left[A_{i}, B_{j}\right]
$$

given by equation (15) gives $X_{1} \rightarrow X$, which is exactly $r N_{1}$-to-one off the locus $x_{1}=0$. The ideal $I \subset R$ generated by the $v$-leading forms of the splice equations includes the forms $x_{i}^{n_{i}}+a_{i} x_{\sigma}^{n_{\sigma}}, 1 \leq i \leq \sigma-1$. Let $J \subset R_{1}$ be the ideal generated by the proper transforms of the elements of $I$ (ie, factor out by the highest power of $A_{1}$ occurring in any equation). Then the induced map $R / I \rightarrow R_{1} / J$ gives a map on spectra which is surjective off the image of the locus $x_{1}=0$. But the $v$-associated graded $R / I$ defines a reduced twodimensional complete intersection, and $x_{1}=0$ is a curve on it (Theorem 2.6); thus, $R / I \rightarrow R_{1} / J$ must be injective.

Next, assign " $v$ '-weights" to the variables of $R_{1}$ :

$$
v^{\prime}\left(A_{1}\right)=\ell_{v v^{*}} / N ; \quad v^{\prime}\left(A_{i}\right)=0, i>1 ; \quad v^{\prime}\left(B_{j}\right)=r \ell_{v w_{j}}-\ell_{v^{*} w_{j}}^{\prime} \ell_{v v^{*}},
$$

where $w_{j}$ is the leaf corresponding to $y_{j}$ or $B_{j}$. It is easy to check that under $R \rightarrow R_{1}, v$-weights give $r$ times $v^{\prime}$-weights. The same is true for the graded injection $R / I \rightarrow R_{1} / J$. 
Finally, the variety corresponding to $J$ has the equations

$$
\begin{aligned}
1+a_{1} A_{\sigma}^{n_{\sigma}} & =0 \\
A_{i}^{n_{i}}+a_{i} A_{\sigma}^{n_{\sigma}} & =0, i \geq 2,
\end{aligned}
$$

which define $N_{1}$ reduced points $\bar{c}=\left(c_{2}, \ldots, c_{\sigma}\right)$. Thus, $R_{1} / J$ is a direct sum of $N_{1}$ graded quotients $R_{1} / J_{\bar{c}}$, where $J_{\bar{c}}$ is obtained by replacing $A_{i}$ by $c_{i}$ (for $i>1$ ) in the defining equations of $J$. It follows as in the discussion of 4.2 that each such quotient is the associated graded of the $\Delta^{\prime}$-splice diagram singularity corresponding to the node $v$ (or equivalently the weight $v^{\prime}$ ) at the point of $X_{1}$ corresponding to $\bar{c}$, ie, at the point $A_{1}=B_{j}=0$ (all $\left.j\right)$ and $A_{i}=c_{i}(i \geq 2)$. This completes the proof of the lemma.

\section{Naturalness of splice diagram equations}

The definition of splice type equations in Theorem 7.2 might appear to depend on the choice of monomials satisfying the relevant conditions, but in fact it does not: for a given node $v$ and edge $e$ at $v$, any two such choices of monomial differ by something of higher order, which can then be absorbed in the higher order terms of the splice diagram equation. Precisely:

Theorem 10.1 Suppose $M=M_{v e}$ and $M^{\prime}=M_{v e}^{\prime}$ are two admissible monomials for $\Delta$ that satisfy the $D(\Gamma)$-equivariance condition. Then for some $a \in \mathbb{C}^{*}, M^{\prime}-a M$ has $v$-weight greater than $d_{v}$. In particular, the corresponding notions of splice diagram equations are the same.

Proof Choose splice diagram equations as in Theorem 7.2 let $(X, o)$ be the resulting complete intersection singularity, and let $Y=X / D(\Gamma)$. Thus $Y$ has a good resolution $\bar{Y}$ with dual graph $\Gamma$. Denote $d=\operatorname{det}(\Gamma)=|D(\Gamma)|$

An analytic function on $Y$ is simply a $D(\Gamma)$-invariant function on $X$, and thus has a $v$-weight for each node of $\Delta$. It also induces a function on the resolution $\bar{Y}$, and thus has an order of vanishing on the exceptional curve $E_{v}$ corresponding to $v$. We recall from item (15) of Theorem 7.2 that, for any node $v$, the $v$-weight of a function $f$ on $Y$ is $d$ times its order of vanishing on the corresponding exceptional curve $E_{v}$ of the resolution.

Now let $E_{1}, \ldots, E_{\delta}$ be the exceptional curves that intersect $E_{v}$, corresponding to edges $e_{1}, \ldots, e_{\delta}$ at $v$. Choose an admissible monomial $M_{i}$ that satisfies the $D(\Gamma)$-equivariance condition for each edge $e_{i}$ at $v$. Each $M_{i}^{d}$ is $D(\Gamma)$-invariant 
and hence defined on $\bar{Y}$ and, by the above remark, it vanishes to order $d_{v}$ on the exceptional curve $E_{v}$. In the same way, at an adjacent node $v^{*}$ of the splice diagram as in

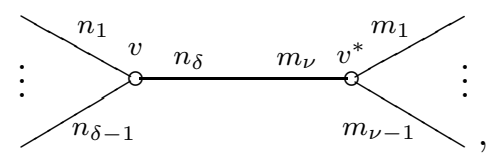

the order of vanishing of $M_{i}^{d}$ on $E_{v^{*}}$ is $d_{v} d_{v^{*}} /\left(n_{\delta} m_{\nu}\right)$ for $i \neq \delta$ and is $n_{\delta} m_{\nu}$ for $i=\delta$. In particular, on $E_{v^{*}}, M_{\delta}^{d}$ vanishes to order $D$ more than the other $M_{i}^{d}$ 's, where $D$ is the edge determinant for the edge $e_{\delta}$.

In the maximal splice diagram we have a node for every exceptional curve and all edge determinants are $d$ (Theorem 12.2). So we have shown that on each $E_{i}$ that intersects $E_{v}$, the $M_{j}^{d}$ with $j \neq i$ vanish to a common order and $M_{i}^{d}$ vanishes to order $d$ greater. Thus $M_{i}^{d} / M_{\delta}^{d}$ on $E_{v}$ has a zero of order $d$ at $E_{v} \cap E_{i}$, a pole of order $d$ at $E_{v} \cap E_{\delta}$, and no other zero or pole.

Let the edge $e$ in the theorem be $e=e_{1}$. Since $M_{1}$ and $M_{\delta}$ transform the same way under $D(\Gamma), M_{1} / M_{\delta}$ is defined on $\bar{Y}$, and $M_{1} / M_{\delta}$ on $E_{v}$ has a simple zero at $E_{v} \cap E_{i}$, a simple pole at $E_{v} \cap E_{\delta}$, and no other poles or zeros. Any other choice $M_{1}^{\prime}$ for $M_{1}$ gives identical zero and pole for $M_{1}^{\prime} / M_{\delta}$. So for some $a \in \mathbb{C}^{*},\left(M_{1}^{\prime}-a M_{1}\right) / M_{\delta}$ vanishes identically on $E_{v}$, whence $\left(M_{1}^{\prime}-a M_{1}\right)^{d}$ vanishes to higher order on $E_{v}$ than does $M_{\delta}^{d}$. Since the $v$-weight of a function $f$ is measured by the order of vanishing of $f^{d}$ on $E_{v}$, the first assertion of the theorem follows.

To prove the second statement of the Theorem, we must show that modulo the equations defining $(X, o), M^{\prime}$ is equal to $a M$ plus monomials of higher $v$-weight. This is the definition of the weight filtration on $X$ (for convenience, we assume that the defining equations are polynomials): if $P$ is the graded polynomial ring in our variables, $I_{k}$ its ideal generated by monomials of $v$ weight $k$, and $J$ the defining ideal for $X$, then the weight filtration on $P / J$ has $k$-th piece $\left(I_{k}+J\right) / J$; so, modulo $J$, anything of weight at least $k$ in $P / J$ can be written as a sum of monomials of degree at least $k$ in the polynomial ring.

\section{Semigroup and congruence conditions in the two- node case}

We first revisit the congruence condition of Proposition 6.8 in the case that the edge $e$ connects $v$ to an end node. Thus suppose we are in the following 
situation, where for convenience in this section we will assume minimal good resolutions (although this is not essential) and $e$ is the edge of $\Delta$ from $v$ to $v^{*}$.

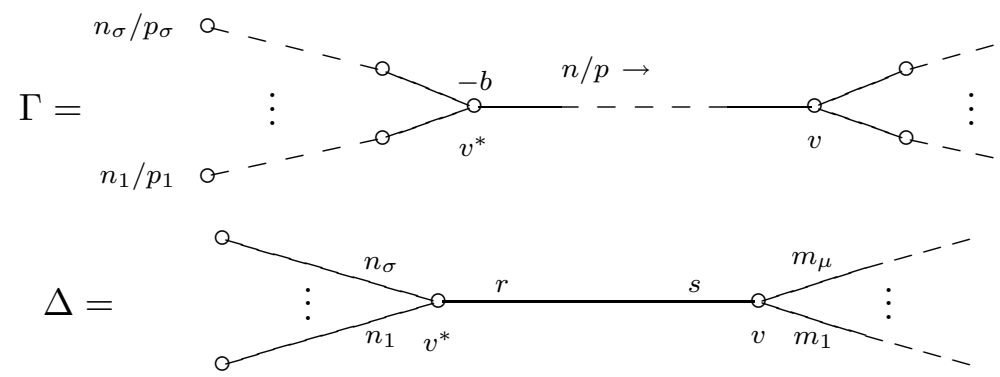

As usual, we represent strings in $\Gamma$ by their continued fractions (the continued fraction for the empty string is $n / p=1 / 0)$. Denote $N=\prod_{i=1}^{\sigma} n_{i}, M=$ $\prod_{j=1}^{\mu} m_{j}, d=\operatorname{det}(\Gamma)$. Then, using Proposition 6.6 the condition of Proposition 6.8 for an admissible monomial $M_{v e}=\prod x_{i}^{\alpha_{i}}$ is that for $i=1, \ldots, \sigma$

$$
\left[\sum_{j \neq i} \alpha_{j} N r /\left(n_{i} n_{j} d\right)+\alpha_{i}\left(N r /\left(n_{i}^{2} d\right)+p_{i}^{\prime} / n_{i}\right)\right]=\left[M N /\left(n_{i} d\right)\right] .
$$

This simplifies to

$$
\left[r s /\left(n_{i} d\right)+\alpha_{i} p_{i}^{\prime} / n_{i}\right]=\left[M N /\left(n_{i} d\right)\right]
$$

or

$$
\left[(r s-M N) /\left(n_{i} d\right)+\alpha_{i} p_{i}^{\prime} / n_{i}\right]=[0] .
$$

Since, by Proposition 12.3 rs $-M N=d n$, the above is equivalent to orequivalently

$$
\begin{aligned}
p_{i}^{\prime} \alpha_{i} \equiv-n \quad\left(\bmod n_{i}\right), \\
\alpha_{i} \equiv-n p_{i} \quad\left(\bmod n_{i}\right) .
\end{aligned}
$$

Now, solutions of these congruences may be written

$$
\alpha_{i}=n_{i}\left\lceil n p_{i} / n_{i}\right\rceil-n p_{i}+n_{i} \delta_{i},
$$

where $\lceil x\rceil$ means least integer $\geq x$; further the non-negativity of the $\alpha_{i}$ is equivalent to the non-negativity of the $\delta_{i}$. Thus the equality $s=\sum \alpha_{i} N / n_{i}$, which expresses that the monomial $M_{v e}$ is admissible, can be written

$$
s=\sum_{i}\left(N\left\lceil n p_{i} / n_{i}\right\rceil-N n p_{i} / n_{i}+N \delta_{i}\right) .
$$

On the other hand, by computing determinant of a star-shaped graph we get

$$
s=N n\left(b-\sum_{i=1}^{\sigma} p_{i} / n_{i}-p / n\right) .
$$


Thus formula (16) is equivalent to

$$
\sum \delta_{i}=n b-p-\sum\left\lceil n p_{i} / n_{i}\right\rceil
$$

If the right hand side of this expression is non-negative, then non-negative $\delta_{i}$ can be found so that the corresponding $\alpha_{i}$ satisfy both the congruence conditions and the semigroup condition at the given edge. We summarize in the

Proposition 11.1 Consider the edge e leading from $v$ to an end-node as above. Then the following inequality is necessary and sufficient in order that the semigroup and congruence conditions are both satisfied for vertex $v$ and edge $e$ :

$$
n b-p-\sum\left\lceil n p_{i} / n_{i}\right\rceil \geq 0 .
$$

This proposition has the immediate corollary:

Proposition 11.2 The following two-node resolution graph (dashed lines represent strings described by continued fractions starting from the interior weights; the central string $n / p$ starts from the left node)

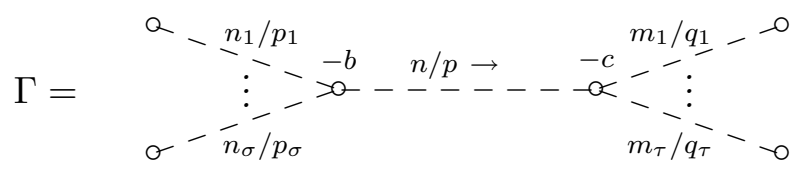

satisfies the semigroup and congruence conditions if and only if

$$
\begin{gathered}
n b-p-\sum\left\lceil n p_{i} / n_{i}\right\rceil \geq 0 \\
n c-p^{\prime}-\sum\left\lceil n q_{j} / m_{j}\right\rceil \geq 0 .
\end{gathered}
$$

We remark that the negative-definiteness of this graph $\Gamma$ is equivalent to the condition that the edge determinant $r s-\prod_{i} n_{i} \prod_{j} m_{j}$ is positive together with the positivity of $s$ and $r$. The latter are slightly weaker conditions than those of the proposition; $s / N>0$ and $r / M>0$ can be written:

$$
\begin{gathered}
s / N=n b-p-\sum n p_{i} / n_{i}>0 \\
r / M=n c-p^{\prime}-\sum n q_{j} / m_{j}>0 .
\end{gathered}
$$

According to our main theorem, $\Gamma$ occurs as the resolution dual graph of a splice-quotient singularity, that is, a surface singularity whose universal abelian cover is of splice type, if the semigroup and congruence conditions are satisfied. We had conjectured earlier that a $\mathbb{Q}$-Gorenstein singularity with $\mathbb{Q} H S$ link is 
always of this type. Although counter-examples are now known (see [5]), the conjecture appears to hold in a surprising number of cases. Singularities with rational or minimally elliptic resolution graphs are automatically $\mathbb{Q}$ - Gorenstein (even Gorenstein for minimally elliptic, 4]), and we assert the truth of the following important

Conjecture 11.3 Let $Y$ be a rational or $\mathbb{Q} H S$-link minimally elliptic surface singularity. Then $Y$ is a splice-quotient singularity as in 7.1 .

Note added April 2005 T Okuma [13] has announced a proof of this conjecture, see Section 13 In the original version of this paper we gave here a partial proof of this conjecture in the two-node case, which we now omit.

\section{Appendix 1: Splicing and plumbing}

This appendix reviews in more detail how a splice diagram is associated to a resolution diagram and explains why it is a topological invariant of the 3 manifold link. We also prove some technical results needed earlier in the paper.

Recall (see the beginning of Section 2) that a splice diagram is a finite tree with no valence 2 vertices, decorated with integer weights as follows: for each node $v$ and edge $e$ incident at $v$ an integer weight $d_{v e}$ is given. Thus an edge joining two nodes has weights associated to each end, while an edge from a node to a leaf has just one weight at the node end. Moreover, we will show that the splice diagrams which arise in the study of links of singularities always satisfy the following conditions:

- All weights are positive.

- All edge determinants are positive.

- The ideal condition (Definition 2.2).

(For the splice diagram associated with an arbitrary graph-manifold rational homology sphere the first two conditions need not hold.) In the process, we will also have need for a variant of splice diagrams where valency 2 vertices are permitted, and weights are also associated to the leaf end of an edge ending in a leaf.

In [1] splice diagrams were used (among other things) to classify the topology of integral homology sphere singularity links. The splice diagrams that arise this way are precisely the splice diagrams as above with pairwise coprime positive weights around each node (in which case the ideal condition is automatic). 
The paper [9] was the first to associate a splice diagram more generally to any rational homology sphere singularity link $\Sigma$. The splice diagram no longer determines the topology of $\Sigma$, but we claim that it does determine the topology of the universal abelian cover of $\Sigma$ (which is $\Sigma$ itself, if $\Sigma$ is a $\mathbb{Z}$-homology sphere). The current paper establishes this assertion only when the semigroup and congruence conditions (2.3 and 6.3) are satisfied, but the result holds without these conditions, and extends even to arbitrary graph-manifold homology spheres; this will be proved elsewhere.

For ease of exposition we restrict to the singularity link case here. In this case we can describe the splice diagram in terms of a resolution of the singularity. This was described briefly at the start of Section [ but we will recall it in more detail.

Thus, let $(Y, o)$ be a normal surface singularity germ and $\Sigma$ its link, that is, the boundary of a regular neighborhood of $o$ in $Y$. Assume that $\Sigma$ is a rational homology sphere, equivalently, $H_{1}(\Sigma)$ is finite. Let $\pi: \bar{Y} \rightarrow Y$ be a good resolution. "Good" means that the exceptional divisor $E=\pi^{-1}(o)$ has only normal crossings. The rational homology sphere condition is equivalent to the condition that $E$ is rationally contractible; that is,

- each component of $E$ is a smooth rational curve;

- the dual resolution graph $\Gamma$ (the graph with a vertex for each component of $E$ and an edge for each intersection of two components) is a tree.

We weight each vertex $v$ of $\Gamma$ by the self-intersection number $E_{v} \cdot E_{v}$ of the corresponding component $E_{v}$ of $E$. The intersection matrix for $\Gamma$ is the matrix $A(\Gamma)$ with entries $a_{v w}=E_{v} \cdot E_{w}$. It is well known that $A(\Gamma)$ is negative-definite and its cokernel (also called the discriminant group) is $H_{1}(\Sigma)$. In particular, $\operatorname{det}(\Gamma):=\operatorname{det}(-A(\Gamma))$ is the order of $H_{1}(\Sigma)$.

A string in $\Gamma$ is a connected subgraph consisting of vertices that have valency $\leq 2$ in $\Gamma$. The resolution is minimal if no $(-1)$-weighted vertex of $\Gamma$ occurs on a string. We do not necessarily want to assume minimality here.

The splice diagram $\Delta$ for $\Sigma$ has the same overall shape as the resolution graph $\Gamma$; it's underlying graph is obtained from $\Gamma$ by suppressing valency two vertices. The weights on edges are computed by the following procedure: At a vertex $v$ of $\Delta$ let $\Gamma_{v e}$ be the subgraph of $\Gamma$ cut off by the edge of $\Gamma$ at $v$ in the direction of $e$, as in the following picture. The corresponding weight is then 
$d_{v e}:=\operatorname{det}\left(\Gamma_{v e}\right)$

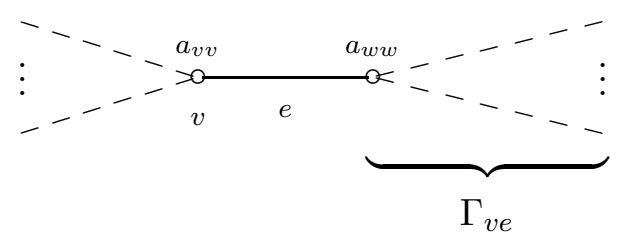

Example 12.1 Here is an example of a resolution graph with integral homology sphere link. The reader can check that $A(\Gamma)$ is negative-definite and unimodular (a quick method is given in [1]).

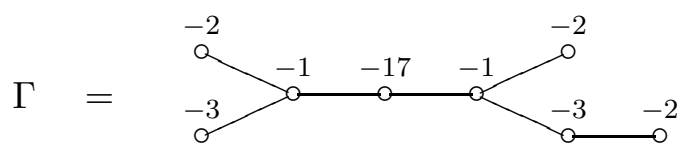

Its splice diagram is:

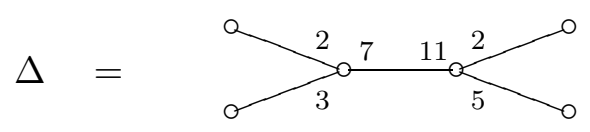

For example, the weight 7 on the left node of $\Delta$ is $\operatorname{det}\left(\Gamma_{v e}\right)$ with

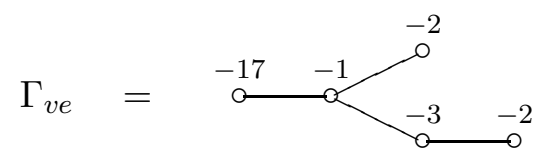

Here is another resolution graph with the same splice diagram

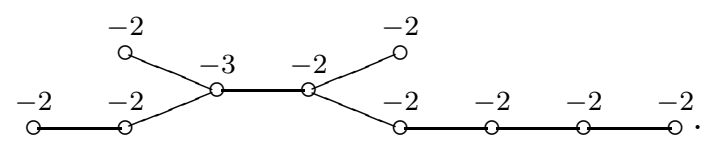

It has discriminant 17 , so its link has first homology $\mathbb{Z} / 17$.

If $\Sigma$ is a $\mathbb{Z}$-homology sphere, then the minimal resolution graph can be recovered from the splice diagram; an algorithm to do this is is described in [11, improving on a procedure in [1]. Thus, in the above example, $\Gamma$ is the only minimal resolution graph with splice diagram $\Delta$ and with $\mathbb{Z}$-homology sphere link. However, there can be several minimal resolution graphs with the same splice diagram representing $\mathbb{Q}$-homology spheres (infinitely many if the splice diagram has just one node and finitely many otherwise).

To understand the resolution graphs that correspond to a given splice diagram it is helpful to consider the maximal splice diagram: the version of the splice 
diagram that we get from the resolution graph if we do not first eliminate vertices of valency 2 , and include edge weights at all vertices - also the leaves. Thus, for the first of Examples 12.1 the maximal splice diagram is:

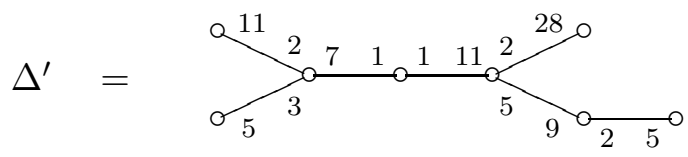

and for the second it is

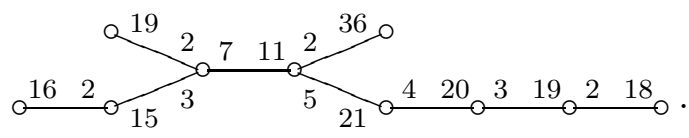

The maximal splice diagram has the following properties.

Theorem 12.2 (1) For any pair of vertices $v$ and $w$ of the maximal diagram let $\ell_{v w}$ be the product of the weights adjacent to, but not on, the shortest path from $v$ to $w$ in $\Delta^{\prime}$ (in particular, $\ell_{v v}=d_{v}$, the product of weights at $v$ ). Then the matrix $L:=\left(\ell_{v w}\right)$ satisfies $\frac{1}{\operatorname{det}(\Gamma)} L=-A(\Gamma)^{-1}$.

(2) Every edge determinant for the maximal splice diagram is $\operatorname{det}(\Gamma)$.

Proof Property (1) of the theorem says

$$
A(\Gamma) L=-\operatorname{det}(\Gamma) I,
$$

which is easily shown by computing that the adjoint matrix of $-A(\Gamma)$ equals $L$. This calculation is carried out explicitly in Lemma 20.2 of [1].

For property (2), suppose we have an edge connecting vertices $v$ and $w$ of the maximal splice diagram as follows,

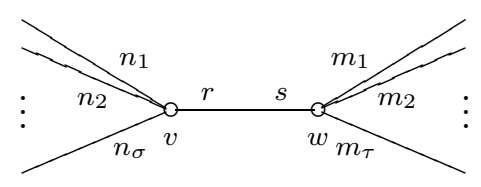

and write $N=\prod_{1}^{\sigma} n_{i}, M=\prod_{1}^{\tau} m_{j}, N_{i}=N / n_{i}$ (if $v$ or $w$ is a leaf the corresponding $N$ or $M$ is 1 ). For each $i=1, \ldots, \sigma$ let $L_{i}$ be the product of the weights just beyond the other end of the $n_{i}$-weighted edge. Then the $v v-$ and $v w$-entries of equation (17) are:

$$
\begin{aligned}
N r a_{v v}+N M+\sum_{1}^{\sigma} r N_{i} L_{i} & =-\operatorname{det}(\Gamma) \\
N M a_{v v}+s M+\sum_{1}^{\sigma} M N_{i} L_{i} & =0 .
\end{aligned}
$$


Multiplying the second of these equations by $r / M$ and then subtracting the first from it gives the desired equation $r s-M N=\operatorname{det}(\Gamma)$.

We can generalize part 2 of the above theorem to any edge of a splice diagram. Let $\Gamma$ be a resolution graph and $\Delta$ its splice diagram. Thus each edge of $\Delta$ corresponds to a string in $\Gamma$.

Proposition 12.3 Let $e$ be an edge of $\Delta$ corresponding to a string $E$ of $\Gamma$. Then the edge determinant $D(e)$ is given by

$$
D(e)=\operatorname{det}(E) \operatorname{det}(\Gamma),
$$

where $\operatorname{det}(E)=1$ if $E$ is the empty string.

Proof We need some preparation.

Lemma 12.4 Suppose in $\Gamma$ we have an extremal string with continued fraction $n / p=b_{1}-1 / \cdots-1 / b_{k}$, and associated splice diagram as follows:

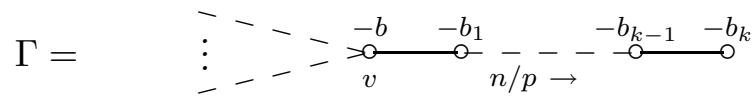

$$
\begin{aligned}
& \Delta=\underbrace{n_{\sigma}}_{n_{1} v} \sigma^{n}
\end{aligned}
$$

Let $\Gamma_{0}$ be the result of removing the string, so it consists of $v$ and what is to the left. Then, with $N=n_{1} \ldots n_{\sigma}$,

$$
\operatorname{det}(\Gamma)=n \operatorname{det}\left(\Gamma_{0}\right)-N p \text {. }
$$

Proof This is the edge determinant equation of part (2) of Theorem 12.2 applied to the edge from $v$ to the $-b_{1}$-weighted vertex in the maximal splice diagram, since the determinant of the string starting at $-b_{2}$ is $p$.

The following lemma has been used earlier (eg, Proposition 6.6), since, even though the edge weight at a leaf is not part of the data of a splice diagram, it is needed in computing discriminant groups. 
Lemma 12.5 Suppose we have a leaf $w$ of a splice diagram

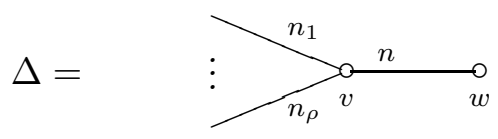

resulting from a resolution string with continued fraction $n / p=b_{1}-1 / \cdots-$ $1 / b_{k}$. Denote by $p^{\prime}$ the discriminant of the string with $w$ removed, so $p p^{\prime} \equiv 1$ (Lemma 6.2, if the string is quasi-minimal then $p^{\prime}$ is the unique such positive integer with $\left.p^{\prime} \leq n\right)$. Denote $N=n_{1} \ldots n_{\sigma}$. Then the splice diagram weight $x$ at $w$ is given by

$$
x=\frac{p^{\prime}}{n} \operatorname{det}(\Gamma)+\frac{N}{n}
$$

Proof Denote $n^{\prime}=\left(p p^{\prime}-1\right) / n$, so $p p^{\prime}-n n^{\prime}=1$. By Lemma 6.2 we have $p^{\prime} / n^{\prime}=b_{1}-1 / \cdots-1 / b_{k-1}$ (if $k=1$, then $\left.p=p^{\prime}=1, n^{\prime}=0\right)$. Apply the previous Lemma 12.4 to both $\Gamma$ and the result $\Gamma^{\prime}$ of removing the rightmost vertex of $\Gamma$. This gives the following (also if $k=1$ ):

$$
\operatorname{det}(\Gamma)=n \operatorname{det}\left(\Gamma_{0}\right)-N p, \quad x:=\operatorname{det}\left(\Gamma^{\prime}\right)=p^{\prime} \operatorname{det}\left(\Gamma_{0}\right)-N n^{\prime} .
$$

Solving the first of these equations for $\operatorname{det}\left(\Gamma_{0}\right)$ and inserting in the second gives

$$
x=p^{\prime}\left(\frac{\operatorname{det}(\Gamma)}{n}+\frac{N p}{n}\right)-N n^{\prime}=\frac{p^{\prime}}{n} \operatorname{det}(\Gamma)+\frac{N}{n},
$$

as desired.

We now complete the proof of Proposition 12.3 by induction on the length of the string. We already know it for the empty string by Theorem 12.2. so suppose we have partially reduced the maximal splice diagram as follows:

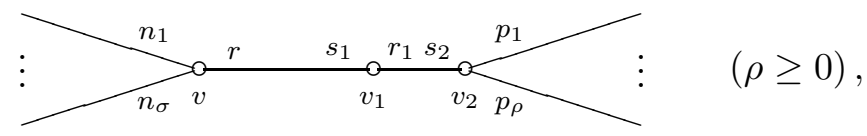

where $v_{1}$ and $v_{2}$ were adjacent in the maximal splice diagram, but the edge from $v$ to $v_{1}$ may correspond to a non-empty string. Denote the string of $\Gamma$ between $v$ and $v_{i}$ by $E_{i}, i=1,2$, and denote $N=n_{1} \ldots, n_{\sigma}, P=p_{1} \ldots, p_{\rho}$.

By Theorem 12.2 and by the induction assumption we have

$$
r_{1} s_{2}-s_{1} P=\operatorname{det}(\Gamma), \quad r s_{1}-N r_{1}=\operatorname{det}\left(E_{1}\right) \operatorname{det}(\Gamma) .
$$

Multiplying the first of these by $N$ and the second by $s_{2}$ and adding gives

$$
s_{1}\left(r s_{2}-N P\right)=\operatorname{det}(\Gamma)\left(s_{2} \operatorname{det}\left(E_{1}\right)+N\right) .
$$


Apply the last lemma (Lemma 12.5) to the result of deleting from $\Gamma$ the vertex $v_{2}$ and all to the right of it. This has determinant $s_{2}$, so Lemma 12.5 gives

$$
s_{1}=\frac{\operatorname{det}\left(E_{1}\right)}{\operatorname{det}\left(E_{2}\right)} s_{2}+\frac{N}{\operatorname{det}\left(E_{2}\right)} \text {. }
$$

Inserting this in the previous equation and simplifying gives

$$
r s_{2}-N P=\operatorname{det}(\Gamma) \operatorname{det}\left(E_{2}\right),
$$

completing the inductive step.

We earlier needed to understand what happens to weights in a splice diagram $\Delta$ when part of the resolution diagram $\Gamma$ changes. Of course weights only change if given by determinants of parts of the resolution graph that have changed. A typical situation might be the following:

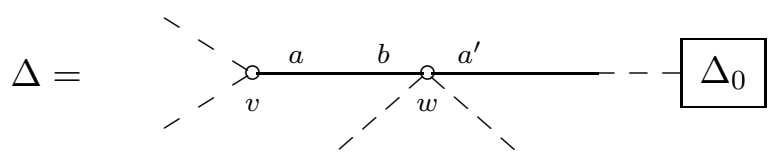

where changing the part of the resolution diagram $\Gamma$ corresponding to $\Delta_{0}$ will not change $b$ but will change the weights $a$ and $a^{\prime}$, say to $\tilde{a}$ and $\tilde{a}^{\prime}$. Denote the changed resolution diagram by $\tilde{\Gamma}$ and the corresponding splice diagram by $\tilde{\Delta}$

Lemma 12.6 Let $M$ be the product of weights other than $a$ at $v$ and $L$ the product of weights other than $b$ and $a^{\prime}$ at $w$.

$$
a \operatorname{det}(\tilde{\Gamma})-\tilde{a} \operatorname{det}(\Gamma)=\frac{M L}{b}\left(a^{\prime} \operatorname{det}(\tilde{\Gamma})-\tilde{a}^{\prime} \operatorname{det}(\Gamma)\right)
$$

Proof Applying Proposition 12.3 to the edge from $v$ to $w$ in $\Delta$ and $\tilde{\Delta}$ gives the equations

$$
\begin{aligned}
& a b-a^{\prime} M L=n \operatorname{det}(\Gamma) \\
& \tilde{a} b-\tilde{a}^{\prime} M L=n \operatorname{det}(\tilde{\Gamma})
\end{aligned}
$$

where $n$ is the determinant of the resolution string for the given edge. Eliminating $n$ from these two equations gives the desired equation.

Lemma 12.7 Suppose that $\Gamma$ is a resolution diagram with a string

$$
\Gamma=\quad->\frac{-b_{0} \frac{-b_{1}}{\longrightarrow}-\infty}{-}-\frac{-b_{n}-b_{n+1}-}{a^{*}}-
$$


and $\tilde{\Gamma}$ results by deleting the node $v^{*}$ at one end of the string and all beyond it:

$$
\tilde{\Gamma}=\quad-\underset{-\infty}{-b_{0}} \stackrel{-b_{1}}{\longrightarrow}--\quad--\stackrel{-b_{n}}{-0}
$$

Suppose $\tilde{\Delta}$ is the corresponding splice diagram, and $a$ and $\tilde{a}$ are the $\Delta$ - and $\tilde{\Delta}$-weights towards $v^{*}$ at a node $v$ of $\tilde{\Gamma}$. Then

$$
a \operatorname{det}(\tilde{\Gamma})-\tilde{a} \operatorname{det}(\Gamma)=M N\left(\ell_{v^{*} v}^{\prime}\right)^{2},
$$

where $M$ is the product of weights of $\Delta$ other than $a$ at $v$ and $N$ is the product of weights of $\Delta$ at $v^{*}$ other than the weight towards $v$.

Proof The result is by induction over the distance from $v^{*}$ to $v$ in $\Delta$. The induction step is the previous lemma. The induction start is the case that $v^{*}$ and $v$ are adjacent in $\Delta$. In this case the equation to be proved can be written $a \operatorname{det}(\tilde{\Gamma})-M N=\tilde{a} \operatorname{det}(\Gamma)$. This is the edge determinant formula of Proposition 12.3 since $\tilde{a}$ is the determinant of the string connecting $v$ to $v^{*}$ in $\Gamma$ and $\operatorname{det}(\tilde{\Gamma})$ is the edge weight of $\Delta$ at $v^{*}$ towards $v$.

\subsection{Topological description of the splice diagram and Ideal Condition}

The weights in a splice diagram have a simple topological meaning. The standard plumbing description (see, eg, [6]) of the manifold $\Sigma=\Sigma(\Gamma)$ associated to a resolution graph (or more general rational plumbing graph) $\Gamma$ shows that to each string in the graph $\Gamma$ is associated an embedded torus in $\Sigma$ such that, if one cuts along these tori, $\Sigma$ decomposes into pieces associated to the leaves and nodes of $\Gamma$. The piece for a leaf is a solid torus, and for a node is of the form (punctured disc) $\times S^{1}$. (If one omits the tori corresponding to leaves, this essentially describes the JSJ decomposition of $\Sigma$.) In particular, the pieces at nodes have natural circle fibers, topologically determined up to isotopy.

Suppose we have a resolution diagram and associated splice diagram as follows:

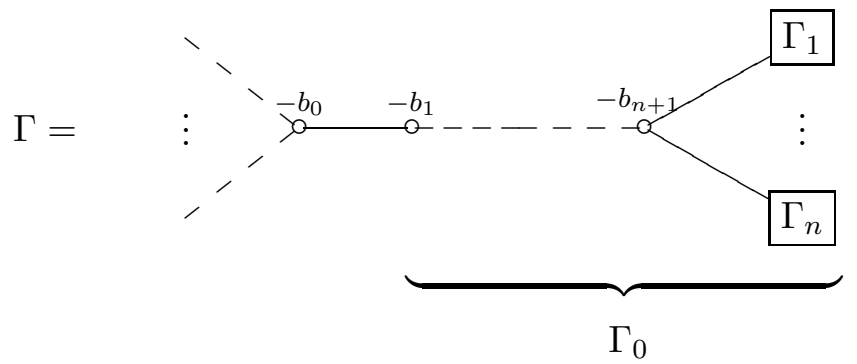




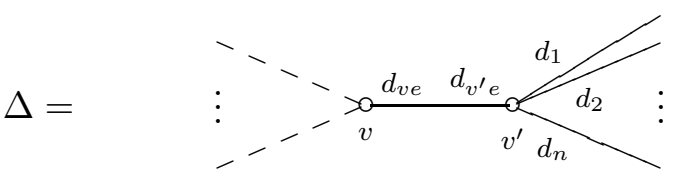

(we are denoting $d_{i}:=d_{v^{\prime} e_{i}}$ for the $i$-th edge $e_{i}$ departing $v^{\prime}$ to the right).

The topological interpretation of $d_{v e}=\operatorname{det}\left(\Gamma_{0}\right)$ is simply that it is the size of $H_{1}\left(\Sigma\left(\Gamma_{0}\right) ; \mathbb{Z}\right)$; topologically $\Sigma\left(\Gamma_{0}\right)$ is the manifold one obtains from the right hand piece after cutting $\Sigma$ along the torus for the edge $e$, by gluing a solid torus into the boundary torus to kill fibers associated to the left node $v$ (ie, match them with meridians of the solid torus).

We can use this for a topological proof that a splice diagram satisfies the ideal condition of Definition 2.2. Recall that it says that for each node $v$ and adjacent edge $e$ of a splice diagram $\Delta$, the edge-weight $d_{v e}$ is in the ideal

$$
\left(\ell_{v w}^{\prime}: w \text { a leaf of } \Delta \text { in } \Delta_{v e}\right) \subset \mathbb{Z} .
$$

Definition 12.8 We will call the positive generator $\bar{d}_{v e}$ of the above ideal the ideal generator for $e$ at $v$. So the ideal condition says $\bar{d}_{v e}$ divides $d_{v e}$.

We refer again to the above diagrams and note that $d_{i}=\left|H_{1}\left(\Sigma\left(\Gamma_{i}\right) ; \mathbb{Z}\right)\right|$ for $i=0, \ldots, n$. Each of the manifolds $\Sigma\left(\Gamma_{i}\right)$ contains a knot $K_{i}$ corresponding to the edge that attaches $\Gamma_{i}$ to the rest of $\Gamma$. Note that the map $H_{1}\left(\Sigma\left(\Gamma_{0}\right) ; \mathbb{Z}\right) \rightarrow$ $H_{1}\left(\Sigma\left(\Gamma_{0}\right) / K_{0} ; \mathbb{Z}\right)$ is surjective, so $\left|H_{1}\left(\Sigma\left(\Gamma_{0}\right) / K_{0} ; \mathbb{Z}\right)\right|$ divides $d_{0}$. The following theorem thus implies the ideal condition.

Theorem 12.9 The ideal generator $\bar{d}_{v e}$ is $\left|H_{1}\left(\Sigma\left(\Gamma_{0}\right) / K_{0} ; \mathbb{Z}\right)\right|$.

We will prove this theorem inductively, so we first describe an inductive computation of the ideal generators.

Lemma 12.10 If $v^{\prime}$ is a leaf $(n=0)$ put $\bar{d}_{v e}=1$. Inductively, if the ideal generator $\bar{d}_{i}$ is known at the $i$-th edge departing $v^{\prime}$ to the right for each $i$ then $\bar{d}_{v e}$ is computed as

$$
\bar{d}_{v e}=\operatorname{gcd}_{i=1}^{n}\left(\bar{d}_{i} \prod_{j \neq i}^{n} d_{j}\right) .
$$

Proof $\bar{d}_{i} \prod_{j \neq i}^{n} d_{j}$ is the generator of the ideal $\left(\ell_{v^{\prime} w}^{\prime}: w\right.$ a leaf of $\Delta$ in $\left.\Delta_{v^{\prime} e_{i}}\right)$, so $\operatorname{gcd}_{i=1}^{n} \bar{d}_{i} \prod_{j \neq i}^{n} d_{j}$ is the generator of the ideal $\left(\ell_{v w}^{\prime}: w\right.$ a leaf of $\Delta$ in $\left.\Delta_{v e}\right)$. 
Proof of Theorem 12.9 Define for the moment $\bar{d}_{i}=\left|H_{1}\left(\Sigma\left(\Gamma_{i}\right) / K_{i} ; \mathbb{Z}\right)\right|$ for each $i$. We will show that these numbers satisfy the inductive formula of the lemma, so they are the ideal generators.

Let $\Delta_{i}$ be the subgraph of the splice diagram $\Delta$ corresponding to the subgraph $\Gamma_{i}$ of $\Gamma . \Sigma=\Sigma(\Gamma)$ contains tori $T_{i}$ corresponding to the edges of $\Delta$ that cut off the subdiagrams $\Delta_{i}$. The torus $T_{0}$ cuts $\Sigma$ into two pieces. We denote the piece corresponding to $\Delta_{0}$ by $\Sigma_{0}^{\prime}$. Thus $\Sigma_{0}$ results from $\Sigma_{0}^{\prime}$ by gluing a solid torus into its boundary, so

$$
H_{1}\left(\Sigma_{0} / K_{0}\right)=H_{1}\left(\Sigma_{0}^{\prime} / T_{0}\right)
$$

Define $\Sigma_{i}^{\prime}$ for $i=1, \ldots, n$ similarly, so

$$
H_{1}\left(\Sigma_{i} / K_{i}\right)=H_{1}\left(\Sigma_{i}^{\prime} / T_{i}\right) .
$$

If we cut $\Sigma$ along all the tori $T_{i}, i=0, \ldots, n$, the central piece $\Sigma_{v^{\prime}}$ corresponding to the node $v^{\prime}$ of $\Delta$ is an $S^{1}$-bundle over an $(n+1)$-punctured sphere $S$. Denote a fiber of this bundle by $f$ and the boundary components of $S$ by $q_{0}, \ldots, q_{n}$, considered as curves in $\Sigma_{v^{\prime}} \cong S^{1} \times S \subset \Sigma_{0}^{\prime}$. For $i=1, \ldots, n, \Sigma_{i}$ is obtained from $\Sigma_{i}^{\prime}$ by gluing in a solid torus with meridian curve $f$, so we have

$$
H_{1}\left(\Sigma_{i}\right)=H_{1}\left(\Sigma_{i}^{\prime}\right) /(f),
$$

so $H_{1}\left(\Sigma_{i}^{\prime}\right) /(f)$ has order $d_{i}$. It follows that

$$
H_{1}\left(\Sigma_{0}^{\prime}\right) /(f)=\bigoplus_{i=1}^{n} H_{1}\left(\Sigma_{i}^{\prime}\right) /(f)
$$

has order $d_{1} \ldots d_{n}$.

By definition of $\bar{d}_{i}$, the quotient $H_{1}\left(\Sigma_{i}^{\prime}\right) /\left(f, q_{i}\right)$ has order $\bar{d}_{i}$, so the order of the element $q_{i} \in H_{1}\left(\Sigma_{i}^{\prime}\right) /(f)$ must be $d_{i} / \bar{d}_{i}$. The element $q_{1}+\cdots+q_{n} \in H_{1}\left(\Sigma_{0}^{\prime}\right) /(f)$ hence has order $\operatorname{lcm}\left(d_{1} / \bar{d}_{1}, \ldots, d_{n} / \bar{d}_{n}\right)$.

Now

$$
H_{1}\left(\Sigma_{0}^{\prime} / T_{0}\right)=H_{1}\left(\Sigma_{0}^{\prime}\right) /\left(f, q_{0}\right)=H_{1}\left(\Sigma_{0}^{\prime}\right) /\left(f, q_{1}+\cdots+q_{n}\right),
$$

so this group has order $\left|H_{1}\left(\Sigma_{0}^{\prime}\right) /(f)\right| / \operatorname{lcm}\left(d_{1} / \bar{d}_{1}, \ldots, d_{n} / \bar{d}_{n}\right)$. This equals

$$
d_{1} \ldots d_{n} / \operatorname{lcm}\left(d_{1} / \bar{d}_{1}, \ldots, d_{n} / \bar{d}_{n}\right)=\operatorname{gcd}_{i=1}^{n}\left(\bar{d}_{j} \prod_{j \neq i}^{n} d_{j}\right)
$$

completing the proof.

The ideal generator is also defined if $v$ is a leaf, and the above proof shows that it equals $\left|H_{1}(\Sigma / K ; \mathbb{Z})\right|$ where $K$ is the knot in $\Sigma$ corresponding to the leaf. Thus: 
Corollary 12.11 The order in homology of the knot in $\Sigma$ corresponding to a leaf of a resolution diagram $\Gamma$ is $\operatorname{det}(\Gamma) / \bar{d}$, where $\bar{d}$ is the ideal generator at the corresponding leaf of the splice diagram.

\section{Appendix 2: Okuma's Theorem}

We conjectured (Conjecture 11.3) that rational singularities and $\mathbb{Q}$ HS-link minimally elliptic singularities are splice quotients. In a recent preprint [13] $\mathrm{T}$. Okuma announces, in effect, that this conjecture is correct. A key is an explicit construction of the UAC, à la Esnault-Viehweg, via a sheaf of algebras on the resolution of $(Y, o)$ [12. The preprint [13 is hesitant about whether the complete intersections he constructs there, which he calls Neumann-Wahl systems, are actually splice type. In fact, they are of splice type. He constructs his complete intersections under a strong condition on the graph (Condition 3.4 of [13]). The key point we make is that a weaker condition, 'Condition 3.3,' that he shows this implies, is equivalent to the semigroup and congruence conditions. To clarify the situation we first assume this equivalence and give versions of Okuma's main results in our language.

Recall that an end-curve on a resolution is a rational curve that has just one intersection point with the rest of the exceptional divisor, so it corresponds to a leaf of the resolution graph.

Theorem 13.1 (Okuma, 13]) Let $(Y, o)$ be a normal surface singularity with $\mathbb{Q} H S$ link whose resolution graph $\Gamma$ satisfies Okuma's 'Condition 3.4', and $\bar{Y} \rightarrow$ $Y$ its minimal good resolution. Suppose that for each end curve $E_{i}$ on $\bar{Y}$ there exists a function $y_{i}: Y \rightarrow \mathbb{C}$ such that the proper transform on $\bar{Y}$ of its zero-locus consists of one smooth irreducible curve $C_{i}$, which intersects $E_{i}$ transversally at one point and intersects no other exceptional curve. Then $(Y, o)$ is a splice-quotient.

We describe 'Condition 3.4' later; for now it suffices that it is stronger than 'Condition 3.3' (ie, the semigroup and congruence conditions). Since it is rarely satisfied for splice-quotients, one would prefer to replace it in the Theorem by the semigroup and congruence conditions. We have even conjectured that the existence of functions $y_{i}$ as above is by itself equivalent to $(Y, o)$ being a splicequotient. This is proved in [11] (Theorem 4.1) when the link is a $\mathbb{Z}$-homology sphere; in this case, the semigroup condition is deduced directly (and no UAC need be constructed). But this conjecture is still open in general. 
Sketch of Proof of Theorem 13.1 Let $d_{i}$ be the order of vanishing of $y_{i}$ on $C_{i}$. Then the "Riemann surface" of $y_{i}^{1 / d_{i}}$ (ie, adjoin the $d_{i}$-th root of $y_{i}$ and normalize) is an abelian cover that is unramified away from the singular point. Thus, a $d_{i}$-th root $z_{i}$ of $y_{i}$ is well defined on the universal abelian cover $(X, o)$ of $(Y, o)$. If the leaves of $\Gamma$ are numbered $i=1, \ldots, t$, we want to show that the $z_{i}, i=1, \ldots, t$, embed $X$ in $\mathbb{C}^{t}$ as a complete intersection of splice type and $Y$ is a splice-quotient of $X$.

Let us verify that the discriminant group $H_{1}(\Sigma)$ acts as specified in Proposition 5.3 on $\mathbb{C}^{t}$. If $\pi: \bar{Y} \rightarrow Y$ is the resolution, then, since the zero-divisor of $y_{i} \circ \pi$ has zero intersection with each $E_{j}$, this divisor is

$$
d_{i} C_{i}-d_{i} \sum_{j} \bar{a}_{i j} E_{j}
$$

where $\left(\bar{a}_{i j}\right)$ is the inverse of the intersection matrix $\left(a_{i j}\right)=\left(E_{i} \cdot E_{j}\right)$. In other words, the order of vanishing of $y_{i}$ along $E_{j}$ is $-d_{i} \bar{a}_{i j}$. Thus the $d_{i}$-th root $z_{i}$ of $y_{i}$ changes by $\exp \left(-2 \pi i \bar{a}_{i j}\right)$ as we go around a meridian curve of $E_{j}$. But this meridian curve represents the element of the discriminant group corresponding to $-e_{j}$ in the notation of Section 5 (the sign results from the convention for how a fundamental group acts as covering transformations) so its action on $z_{i}$ is indeed as in Proposition 5.3 (see Lemma 6.4).

We next need to know that the $z_{i}$ generate the maximal ideal at our singular point. This is a significant step in Okuma's proof, and is where he needs 'Condition 3.4' (as opposed to simply the semigroup and congruence conditions); we do not attempt to reprove it here.

Since the semigroup and congruence conditions are satisfied by assumption, we can choose a system of admissible monomials in the $z_{i}$ that transform correctly under the discriminant group. Let $M_{1}, \ldots, M_{\delta}$ be the monomials corresponding to the $\delta$ edges at a node $v$ of the splice diagram. Then, as in the proof of Theorem 10.1 (see also the proof of Theorem 4.1 of [11]), the ratios $M_{i} / M_{\delta}$ are invariant under the discriminant group, hence defined on $\bar{Y}$, and each has just a single zero and a single pole on $E_{v}$ (at the intersections with the neighboring curves $E_{v_{i}}$ and $E_{v_{\delta}}$ respectively). It follows that there are $\delta-2$ linear relations among the $M_{i} / M_{\delta}$ up to higher order at $E_{v}$. Multiplying by $M_{\delta}$, we see that the $z_{i}$ satisfy a system of splice type equations, compatible with the discriminant group action. We thus get a map of $(Y, o)$ to the corresponding splice quotient and it is not hard to see it is an isomorphism.

Theorem 13.2 (Okuma, [13]) If $(Y, o)$ is rational or $\mathbb{Q} H S$-link minimally elliptic, then the semigroup and congruence conditions are satisfied and functions $y_{i}$ as in the above theorem exist. In addition, $(Y, o)$ is a splice quotient. 
Proof The existence of functions $y_{i}$ as in Theorem 13.1 is standard in the rational case, and, as Okuma points out, follows also in the minimally elliptic case by the arguments of Miles Reid in [14] (Lemma, p. 112). It thus remains to discuss 'Condition 3.4' and the semigroup and congruence conditions. We first give some of Okuma's terminology.

A $\mathbb{Q}$-cycle is a rational linear combination of the exceptional curves $E_{i}$. For each $i$ Okuma denotes by $\bar{E}_{i}$ the $\mathbb{Q}$-cycle "dual" to $E_{i}$ in the sense that $\bar{E}_{i} \cdot E_{j}=-\delta_{i j}$ (so $\bar{E}_{i}=-e_{i}$ in the notation of Section 51). By Theorem 12.2.

$$
\bar{E}_{i}=\frac{1}{\operatorname{det}(\Gamma)} \sum_{j} \ell_{i j} E_{j}
$$

A monomial cycle is a non-negative integer linear combination

$$
D=\sum_{k \in \mathcal{E}} \alpha_{k} \bar{E}_{k}, \quad \text { where } \mathcal{E}=(\text { the ends of } \Gamma) .
$$

Okuma calls each connected component $C$ of $E-E_{i}$, for any $i$, a branch of $E_{i}$. We denote by $\Gamma_{C}$ the corresponding subgraph of $\Gamma$.

The following is 'Condition 3.4', which is well known for rational and $\mathbb{Q} H S$-link minimally elliptic singularities; as Okuma says, it follows from basic results on computation sequences, eg, 3, 4]). As mentioned above, this condition is at present needed to show the UAC is a complete intersection.

'Condition 3.4' For any branch $C$ of any $E_{i}$ not an end-curve, the fundamental cycle $Z_{C}$ for $\Gamma_{C}$ satisfies $Z_{C} \cdot E_{i}=1$. (The fundamental cycle is the minimal effective cycle that has non-positive intersection number with each $E_{j}$ in $C$.)

Okuma shows this condition implies the following 'Condition 3.3', which we will show is equivalent to the semigroup and congruence conditions:

'Condition 3.3' For any node $i$ of $\Gamma$ and branch $C$ of $E_{i}$ there exists a monomial cycle $D$ such that $D-\bar{E}_{i}$ is an effective integral cycle (ie, with non-negative integral coefficients) supported on $C$. Moreover $D$ has the form $D=\sum_{k} \alpha_{k} \bar{E}_{k}$ with $k$ running only through the leaves of $\Gamma$ in $\Gamma_{C}$.

We have included the second sentence of the condition for convenience; one can show it follows from the first.

Okuma's proof that that 'Condition 3.4' implies 'Condition 3.3' is elegant and simple: We want to add an effective integral cycle to $\bar{E}_{i}$ to get a monomial 
cycle $D$ as in 'Condition 3.3'. Let $C_{1}=C$ be the branch in question and put $D_{1}=\bar{E}_{i}+Z_{C}$. Clearly $D_{1} \cdot E_{j}=0$ for each $j$ outside $\Gamma_{C}$ and $D_{1} \cdot E_{j} \leq 0$ otherwise. If $D_{1} \cdot E_{j}=\alpha<0$ for some $j$ other than a leaf of $\Gamma$ in $\Gamma_{C}$, choose such a $j$ as close as possible to $i$, let $C_{2}$ be a branch of $j$ that is in $C$, and put $D_{2}=D_{1}-\alpha Z_{C_{2}}$. Repeat until you have $D$ with $D \cdot E_{j}=0$ for all $j$ that are not leaves of $\Gamma$ in $\Gamma_{C}$. Then $D=\sum_{k} \alpha_{k} \bar{E}_{k}$ with $\alpha_{k}=-D \cdot E_{k}$ for each $k$ a leaf of $\Gamma$ in $\Gamma_{C}$.

Proposition 13.3 'Condition 3.3' is equivalent to the semigroup and congruence conditions.

Proof Suppose 'Condition 3.3' holds for a node $i$ of $\Gamma$. In the following $k$ runs through the leaves of $\Gamma$ in $\Gamma_{C}$ and $j$ runs through all vertices of $\Gamma$.

$$
\begin{aligned}
\operatorname{det}(\Gamma)\left(D-\bar{E}_{i}\right) & =\sum_{k} \alpha_{k} \sum_{j} \ell_{k j} E_{j}-\sum_{j} \ell_{i j} E_{j} \\
& =\sum_{j}\left(\sum_{k} \alpha_{k} \ell_{k j}-\ell_{i j}\right) E_{j} .
\end{aligned}
$$

Since this cycle is supported in $C$, the coefficient of $E_{i}$ is zero:

$$
\sum_{k} \alpha_{k} \ell_{k i}=\ell_{i i}
$$

This is the semigroup condition. Note that the vanishing of the coefficient of any $E_{j}$ with $E_{j}$ not in $C$ gives a multiple of this equation, so the semigroup condition is equivalent to these coefficients vanishing for all $E_{j}$ not in $C$.

Now look at the coefficient of an $E_{j}$ that is in $C$. The condition that $D-\bar{E}_{i}$ is an integral cycle says

$$
\sum_{k} \alpha_{k} \ell_{k j} \equiv \ell_{i j} \quad(\bmod \operatorname{det}(\Gamma)) .
$$

Comparing with Propositions 6.8 and 6.5 we see that as $j$ runs through leaves of $\Gamma$ this is the congruence condition. Recall that the congruence condition is an equivariance condition and the above confirms this equivariance for the generators of the discriminant group corresponding to leaves of $\Gamma$. The above congruence for other $E_{j}$ in $C$ is the equivariance condition for the group elements corresponding to these vertices, and thus follows once one knows it for the generators. Thus the congruence condition for the given node and branch is equivalent to the above congruence as $E_{j}$ runs through exceptional curves in $C$. 


\section{References}

[1] D Eisenbud, W D Neumann, Three-dimensional link theory and invariants of plane curve singularities, Ann. Math. Stud. 110, Princeton Univ. Press (1985) MathReview

[2] Robin Hartshorne, Algebraic Geometry, Graduate Texts in Mathematics 52, Springer-Verlag (1977) MathReview

[3] H B Laufer, On rational singularities, Amer. J. Math. 94 (1972) 597-608 MathReview

[4] H B Laufer, On minimally elliptic singularities, Amer. J. Math. 99 (1977) 1257-1295 MathReview

[5] I Luengo-Velasco, A Melle-Hernandez, A Némethi, Links and analytic invariants of superisolated singularities, to appear in J. Alg. Geom. arXiv:math.AG/0312416

[6] W D Neumann, A calculus for plumbing applied to the topology of complex surface singularities and degenerating complex curves, Trans. Amer. Math. Soc. 268 (1981) 299-343 MathReview

[7] W D Neumann, Abelian covers of quasihomogeneous surface singularities, from: "Singularities, Arcata 1981", Proc. Symp. Pure Math. 40 Amer. Math. Soc. (1983) 233-243 MathReview

[8] W D Neumann, G A Swarup, Canonical decompositions of 3-manifolds, Geom. Topol. 1 (1997) 21-40 MathReview

[9] W D Neumann, J Wahl, Universal abelian covers of surface singularities, from: "Trends in Singularities", (A Libgober and M Tibar, editors) Trends Math. Birkhäuser (2002) 181-190 MathReview

[10] W D Neumann, J Wahl, Universal abelian covers of quotient-cusps, Math. Ann. 326 (2003) 75-93 MathReview

[11] W D Neumann, J Wahl, Complex surface singularities with integral homology sphere links, Geom. Topol. 9 (2005) 757-811

[12] T Okuma, Universal abelian covers of rational surface singularities, J. London Math. Soc. 70 (2004) 307-324 MathReview

[13] T Okuma, Universal abelian covers of certain surface singularities, arXiv:math.AG/0503733

[14] M Reid, Chapters on Algebraic Surfaces, from: "Complex algebraic geometry (Park City, UT, 1993)", IAS/Park City Math. Ser. 3, Amer. Math. Soc. (1997) 3-159 MathReview 